Review

\title{
Key to Xenobiotic Carotenoids
}

\author{
Hans-Richard Sliwka * and Vassilia Partali \\ Department of Chemistry, Norwegian University of Science and Technology, 7491 Trondheim, \\ Norway
}

* Author to whom correspondence should be addressed; E-Mail: richard.sliwka@ntnu.no;

Tel.: +47-73595600; Fax: +47-73596255.

Received: 9 January 2012; in revised form: 21 February 2012 / Accepted: 22 February 2012 /

Published: 7 March 2012

\begin{abstract}
A listing of carotenoids with heteroatoms (X = F, Cl, Br, I, Si, N, S, Se, Fe) directly attached to the carotenoid carbon skeleton has been compiled. The 178 listed carotenoids with $\mathrm{C}, \mathrm{H}, \mathrm{X}$ atoms demonstrate that the classical division of carotenoids into hydrocarbon carotenoids $(\mathrm{C}, \mathrm{H})$ and xanthophylls $(\mathrm{C}, \mathrm{H}, \mathrm{O})$ has become obsolete.
\end{abstract}

Keywords: carotenoids; xanthophylls; fluorocarotenoids; chlorocarotenoids; bromocarotenoids; iodocarotenoids; silicon carotenoids; nitrogen carotenoids; sulfur carotenoids; selenium carotenoids; iron carotenoids

\section{Introduction}

The number of natural occurring carotenoids registered in the relevant books on the topic has increased continuously: 19 carotenoids in 1934, 67 in 1948, 273 in 1971, 563 in 1987, 750 in 2004 [1-5]. The importance of the Carotenoids Handbook is evident for all those working frequently or occasionally with carotenoids. However, the extensive compilation of natural occurring carotenoids has ignored the existence of the numerous xenobiotic carotenoids [6]. The impact of the Carotenoids Handbook is overwhelming insofar that carotenoids with atoms other than $\mathrm{C}, \mathrm{H}, \mathrm{O}$ are barely thinkable. Carotenoids are still classified in two groups: carotenes (polyenes containing $\mathrm{C}, \mathrm{H}$ ) and xanthophylls (polyenes with $\mathrm{C}, \mathrm{H}, \mathrm{O}$ ), and the occurrence of carotenoids with other atoms was not contemplated by the existing nomenclature rules. In contrast, the Natural Product Reports dedicate a specific chapter to steroids with heteroelements, sulfur flavonoids and heteroatom-substituted carbohydrates have been reviewed. [7-10]. Admittedly, no hetero-carotenoids have been detected so far in Nature, but 
nonetheless, it is not incongruous to expect carotenoids from sea organisms to incorporate $\mathrm{Cl}$ (compounds $\mathbf{5 C l - 8 C l}$ in the list) [11]; the interactions between selenium and carotenoids support speculations about the existence of combination products [12-14]. After all, heterocarotenoids may not keep forever their status as xenobiotic compounds, though by then, xenophobia towards xenobiotic carotenoids may be encountered. In a historical review on the "Development of Carotenoid Chemistry 1922-1991" the first Br-, N- and S-carotenoids (4Br-9Br, 2N, 12S) were ignored [15]. When the author's first manuscript on carotenoid thioketones (1S-3S) was rejected by the referees, the honorary co-author commented the rejection as the logical consequence of working with bizarre compounds. The syntheses of selenium carotenoids (1Se-7Se) were regarded by some of the author's colleagues as a completely useless, ill-famed and ill-smelling occupation. Strangely enough, the summarizing speaker at the end of a carotenoid conference intentionally omitted to mention the author's presentation on $\mathrm{S}, \mathrm{N}$ and Se carotenoids. Fortunately, these narrow-minded discriminatory prejudices have now tended to cease; heterocarotenoids have found applications impossible to achieve with "normal" carotenoids, e.g., 2S, 15S, 3Se, 12N, 46N [16-19].

Despite the increasing interest in xenobiotic carotenoids, searching the databases for these compounds often results in zero hits. The unawareness of heterocarotenoids may perhaps be the reason for avoidable syntheses. The molecular wire carotenoid thiol $\mathbf{1 5 S}$ was prepared in several steps [16]. Carotenoid thione 2S, synthesized previously from a commercial carotenoid in a one-step synthesis, could probably have been more appropriate for the investigation [20]. Even an author sensitized to xenobiotic carotenoids witnessed ignorance; compounds $25 \mathrm{~N}, 27 \mathrm{~N}, 29 \mathrm{~N}$ were not cited in a paper on carotenoid oxime hydrochlorides $\mathbf{1 9 N}-\mathbf{2 2 N}[21,22]$. Unfamiliarity with heterocarotenoids is possibly the cause for further lack of mention, e.g., nitrile carotenoid $\mathbf{6 N}$ was patented in 1990 and published in 2011 without referring to previous work from 1988; thienyl carotenoid 3OS, first reported in 1981, was not cited when the compound was resynthesized 20 years later (for an explanation of the designation 3OS see Section 4: Nomenclature).

This thematic issue of Molecules on "Carotenoids" now offers the opportunity to compile a systematic listing of xenobiotic carotenoids. This inventory is a first attempt to take these carotenoids out of their obscurity.

\section{Historical Remarks}

Carotenoids became eye-catching in 1906 with the invention of chromatography by Tswett and got scientific consecration with the first determination of their molecular formula by Willstäter in 1907 $[23,24]$. During the period of structure determination the first nitrogen carotenoids were prepared as analytical derivatives (oxime, semicarbazone) [25,26]. Bromo and sulfur carotenoids were synthesized in 1958 and 1959 and chloro carotenoids in 1976 [27-29]. The synthesis of carotenoid amines was not successful until 1990 [30-32]. The most heterogenic carotenoids are probably iron carbonyl compounds 5Fe-7Fe. The common Greek-letter termed cyclic end groups are now increasingly being replaced by heterocycles. 


\section{Selection Criteria}

Polyenes with a branched polyene chain $>\mathrm{C} 20$ capped with different cyclic or acyclic end groups and with heteroatoms covalently bound to the carbon carotenoid were considered. Thus, compounds with a heteroatom linked via oxygen to the carotenoid scaffold were omitted (e.g., phosphates). Adhering strictly to the isoprenoid nature of carotenoids would not allow including the interesting aza compound 37N [33]. This compound has been perceived as an azine of retinal, but is much more attractive when viewed as a diazapolyene. Various carotenoid derivatives prepared for analytical purposes (oximes, hydrazones amides etc.) are not mentioned [34,35], unless the derivative has also found an application extending characterization, e.g., canthaxanthin oxime was skipped, canthaxanthin oxime hydrochloride $\mathbf{2 1} \mathbf{N}$ as a surface active hydrophilic carotenoid was included [22]. Some carotenoids are drawn in the concise all-trans form, since the dimension of the actual cis-isomers would be too space demanding, e.g., $32 \mathrm{~N}$ and $33 \mathbf{N}$. The main concern of the recording, the heteroatom character of the compound, is not affected by this presentation.

In a departure from Molecules' normal style, reference registration in the compound list follows the example of the handbooks excluding article title and search-irrelevant data on the length of a paper. The references for the individual compounds are not exhaustive. A reader interested in a particular compound should perform a structural search in a database to receive complete and updated citations.

There certainly exist more xenobiotic carotenoids than presented in the list. Many hetero-carotenoids, especially from the patent literature, are not recorded owing to search problems or involuntary neglect. Such compounds ought to be included in a forthcoming extended register. Enlarging the selection criteria to $<\mathrm{C} 20$ chains, to xenobiotic $\mathrm{C}, \mathrm{H}, \mathrm{O}$ carotenoids, considering heteroatoms outside the carotenoid carbon skeleton sphere and taking into account ionic bounded heteroatoms is desirable for future compilation [36,37]. It would furthermore be valuable to have at hand a complete directory of isotope-substituted carotenoids (D, T, ${ }^{13} \mathrm{C},{ }^{14} \mathrm{C}$ ) [38-40]. A catalog of modified carotenoids (e.g., long chain carotenoids, carotenoid dimers, carotenoids with deviated conjugation, hydrophilic carotenoids) and of compounds where carotenoids are part of other molecule classes (e.g., carotenoid lipids, antioxidant combinations) would likewise be desirable [41-51].

\section{Nomenclature}

The designation "xenobiotic carotenoids" is synonymously used with the term "heterocarotenoids". Whereas "heterocarotenoids" may appear more precise, the prefix hetero- is too strongly linked with heterocyclic chemistry and could create confusing expressions such as heterocyclic heterocarotenoids. Xenobiotic is, at present, the more explanatory designation.

Applying the nomenclature rules to xenobiotic carotenoids can lead to unintelligible descriptions; consequently, many authors have avoided naming their products, e.g., 11Cl-15Cl [52]. Keeping in mind that a short trivial name engenders more associative information than a (semi)systematic designation, some names in the list may appear randomly chosen or meaningless. A name search in a database will, therefore, often be unsuccessful, e.g., the name dicyano-C48:15 for $\mathbf{9 N}$ is certainly not canonical, but articulates the essential information: a dicyano substituted carotenoid of $48 \mathrm{C}$ with 15 conjugated double bonds. The exact name would hide this evidence. In any case, the interested 
reader should certainly scrutinize the carotenoids visually and not by their appellation, and a structure search in a database is, therefore, recommended. The structures are approximately listed according to increasing structural complexity; however, the relation to a parent compound was considered more important than complexity ranking.

Aryl carotenoids have been recorded separately within a heteroatom section. Natural occurring aryl carotenoids display trimethylbenzene $\phi-$ or $\chi$ - end groups. Phenyl end groups without methyl are identified as either 16,17,18-trinor- $\phi-$ or 16,17,18-trinor- $\chi-$; nevertheless, the letter $\phi$ is preferred, in analogy to the widely used short form of $\phi$ for phenyl [53]. Thus, all carotenoids with a benzene ring are termed $\phi$-carotenoids; the $\phi$-ring positions are indicated as recommended by the nomenclature rule.

The compounds were arbitrarily numbered; the numbers are not intended to reflect the appointed personal identification digits used in the Key to Carotenoids and the Carotenoids Handbook [4,5]. Carotenoids with heterocycles were, for example, enumerated as $\mathrm{x} \odot \mathrm{S}, \odot \mathrm{S}$ indicating a cycle with sulfur.

The catalog of xenobiotic carotenoids definitely proves that the term xanthophyll has become obsolete [54]. Applying the classical two level differentiation - hydrocarbon carotenoids $(\mathrm{C}, \mathrm{H})$ and xanthophylls $(\mathrm{C}, \mathrm{H}, \mathrm{O})$ - simply implies denying the existence of the listed 178 carotenoids. The use of xanthophyll is therefore discouraged and should be replaced by oxygen carotenoids; such a designation is unequivocally extendable to sulfur (nitrogen, halogen ...) carotenoids.

\section{Conclusions}

Xenobiotic carotenoids have been synthesized for a long time but have remained largely unnoticed by carotenoid chemists. Many of those who work with these compounds may not consider themselves carotenoid chemists. Heteroatoms have helped carotenoids to leave their terrain of origin: xenobiotic carotenoids merit the same appreciation as biotic carotenoids.

\section{Acknowledgement}

The authors thank a reviewer for a reference to additional sulfur carotenoids.

\section{References}

1. Zechmeister, L. Carotenoide; Springer: Berlin, Germany, 1934.

2. Karrer, P.; Jucker, E. Carotenoide; Birkhäuser: Basel, Switzerland, 1948.

3. Karrer, P.; Jucker, E. Carotenoids; Elsevier: Amsterdam, The Netherlands, 1950.

4. Straub, O.; Pfander, H. Key to Carotenoids; Birkhäuser: Basel, Switzerland, 1987.

5. Britton, G., Liaaen-Jensen, S., Pfander, H., Eds. Carotenoids Handbook; Birkhäuser: Basel, Switzerland, 2004.

6. IUPAC. "A xenobiotic is a compound that is foreign to a living organism". Glossary of terms used in bioinorganic chemistry (IUPAC Recommendations). Pure Appl. Chem. 1997, 69, 1303.

7. Levine, P.A.; Sobotka, H. The thio-sugar from yeast. J. Biol. Chem. 1925, 65, 551-554.

8. Kocourek, J.; Klenha, J.; Jiracek, V. Preparation of 1-seleno-1-deoxy-D-glucose. Chem. Ind. 1963, 1397-1397. 
9. Ohle, H.; Lichtenstein, R. Über die Aceton-Verbindungen der Zucker und ihrer Derivate XVII: Umwandlung der Monoaceton-Glucose in eine neue Amino- und Anhydro-Hexose. Ber. Dtsch. Chem. Ges. 1930, 64, 2905-2912.

10. Balint, R.; Bognar, R.; Rakoski, M. Chemistry of sulfur containing flavonoids. In Organic Sulfur Chemistry: Theoretical and Experimental Advances; Bernardi, F., Csizmadia, I.G., Mangini, A., Eds.; Elsevier: Amsterdam, The Netherlands, 1985; pp. 660-706.

11. Gribble, G.W. Naturally-occurring organohalogen compounds-A survey. J. Nat. Prod. 1992, 55, 1353-1395.

12. Neamțu, G.; Bodea, C. Chemotaxonomy of higher plants 1. Carotinoid pigments in genus Astragalus. Rev. Roum. Biochim. 1969, 6, 157-161.

13. Krief, A.; Hevesi, L. Organoselenium Chemistry; Springer: Berlin, Germany, 1988.

14. Biacs, P.A.; Daood, H.G.; Kadar, I. Effect of Mo, Se, Zn, and Cr Treatments on the yield, element concentration, and carotenoid content of carrot. J. Agric. Food Chem. 1995, 43, 589-591.

15. Eugster, C.H. Die Entwicklung der Carotinoid-Chemie im Spiegel der Helvetica Chimica Acta 1922-1991. Helv. Chim. Acta 1992, 75, 941-994.

16. Sliwka, H.R. Conformation and circular dichroism of $\beta, \beta$-carotene derivatives with nitrogen-, sulfur-, and selenium-containing substituents. Helv.Chim. Acta 1999, 82, 161-169.

17. Leatherman, G.; Durantini, E.N.; Gust, D.; Moore, T.A.; Moore, A.L.; Stone, S.; Zhou, Z.; Rez, P.; Liu, Y.Z.; Lindsay, S.M. Carotene as a molecular wire: Conducting atomic force microscopy. J. Phys. Chem. B 1999, 103, 4006-4010.

18. Ion, A.; Partali, V.; Sliwka, H.R.; Banica, F.G. Electrochemistry of a carotenoid self-assembled monolayer. Electrochem. Commun. 2002, 4, 674-678.

19. Mohamad, S.B.B.; Yousef, Y.A.; Melø, T.B.; Javorfi, T.; Partali, V.; Sliwka, H.R.; Naqvi, K.R. Singlet oxygen quenching by thione analogues of canthaxanthin, echinenone and rhodoxanthin. J. Photochem. Photobiol. B Biol. 2006, 84, 135-140.

20. Sliwka, H.R.; Liaaen-Jensen, S. Synthetic sulfur carotenoids 3. Carotenoid thiones $1^{\text {st }}$ preparation and spectroscopic properties. Acta Chem. Scand. 1994, 48, 679-683.

21. Moore, T.A.; Song, P.S. Electronic-spectra of carotenoids-Schiff's bases of carotenal and carotenones. J. Mol. Spec. 1974, 52, 224-232.

22. Willibald, J.; Rennebaum, S.; Breukers, S.; Abdel Hafez, S.H.; Patel, A.; Øpstad, C.L.; Schmid, R.; Nalum Naess, S.; Sliwka, H.R.; Partali, V. Hydrophilic carotenoids: Facile syntheses of carotenoid oxime hydrochlorides as long-chain, highly unsaturated cationic (bola)amphiphiles. Chem. Phys. Lipids 2009, 161, 32-37.

23. Tswett, M. Physikalisch-chemische Studien über das Chlorophyll. Ber. Dtsch. Botan. Ges. 1906, 24, 316-323.

24. Willstätter, R.; Mieg, W. Über eine Methode der Trennung und Bestimmung von Chlorophyllderivaten. Liebigs Ann. Chem. 1906, 350, 1-47.

25. Kuhn, R.; Grundmann, C. Die Konstitution des Lycopins. Ber. Dtsch. Chem. Ges. 1932, 65B, 1880-1889.

26. Karrer, P.; Solmssen, U.; Gugelmann, W. $\beta$-Apo-4-carotinal, ein weiteres Abbauproduckt des $\beta$ - Carotins. Helv. Chim. Acta 1937, 20, 1020-1024. 
27. Entschel, R.; Karrer, P. Carotinoidsynthesen 22. Umsetzungsprodukte des $\beta$-Carotins mit Bromsuccinimid-(Einführung von Äther-Gruppen und Hydroxyl-Gruppen in den Kohlenwasserstoff). Helv. Chim. Acta 1958, 41, 402-414.

28. Martin, C.; Karrer, P. Carotinoidsynthesen 26. Einführung von Mercapto-Gruppen und AnilidoGruppen in das $\beta$-Carotin. Helv. Chim. Acta 1959, 42, 464-466.

29. Pfander, H.; Leuenberger, U. Chlorinated carotenoids from $\mathrm{CHCl}_{3}-\mathrm{HCl}$-reaction. Chimia 1976, 30, 71-73.

30. Petracek, F.J.; Zechmeister, L. The Hydrolytic cleavage products of boron trifluoride complexes of $\beta$-carotene, some dehydrogenated carotenes and anhydrovitamin-A. J. Am. Chem. Soc. 1956, 78, 3188-3191.

31. Bush, W.V.; Zechmeister, L. On some cleavage products of the boron trifluoride complexes of $\alpha$ carotene, lycopene and $\gamma$-carotene. J. Am. Chem. Soc. 1958, 80, 2991-2999.

32. Inanaga, J.; Yamaguchi, M. Transformation of zeaxanthin into 3,3'-diamino- and dimercapto- $\beta$ carotene derivatives. Mem. Fac. Sci. Kyushu Univ. Ser. C 1989, 17, 109-112.

33. Weedon, B.C.L.; Moss, G.P. Structure and nomenclature. In Carotenoids; Britton, G., Liaaen-Jensen, S., Pfander, H., Eds.; Birkhäuser: Basel, Switzerland, 1995; Volume 1A, p. 28.

34. Liaaen-Jensen, S. Isolation, reactions. In Carotenoids; Isler, O., Ed.; Birkhäuser: Basel, Switzerland, 1971; p. 87.

35. Winterstein, A.; Studer, A.; Ruegg, R. Neuere Ergebnisse der Carotinoidforschung. Chem. Ber. 1960, 93, 2951-2965.

36. Dirks, G.; Moore, A.L.; Moore, T.A.; Gust, D. Light-absorption and energy-transfer in polyeneporphyrin esters. Photochem. Photobiol. 1980, 32, 277-280.

37. Rudatsikira, A.; Francois, B.; Mathis, C. Electron-transfer from carbanionic salts to linear sequences of conjugated double-bonds 2 . Reaction with trans- $\beta$-carotene. Makromol. Chem. 1989, 190, 93-105.

38. Liebman, A.A.; Burger, W.; Choudhry, S.C.; Cupano, J. Synthesis of carotenoids specifically labeled with isotopic carbon and tritium. Methods Enzymol. 1992, 213, 42-49.

39. Lugtenburg, J.; Creemers, A.F.L.; Verhoeven, M.A.; van Wijk, A.A.C.; Verdegem, P.J.E.; Monnee, M.C.F.; Jansen, F.J.H.M. Synthesis of ${ }^{13} \mathrm{C}$-labeled carotenoids and retinoids. Pure Appl. Chem. 1999, 71, 2245-2251.

40. Eguchi, T.; Dekishima, Y.; Aizawa, H.; Tamegai, H.; Kakinuma, K.; Misawa, N.; Kuzuyama, T.; Seto, H. Preparation of highly deuterated zeaxanthin, lycopene, and $\beta$-carotene from fully deuterated mevalonate using engineered Escherichia coli. Tetrahedron 2005, 61, 2027-2035.

41. Karrer, P.; Eugster, C.H. Carotinoidsynthesen VIII. Synthese des Dodecapreno- $\beta$-Carotins. Helv. Chim. Acta 1951, 34, 1805-1814.

42. Partali, V.; Kvittingen, L.; Sliwka, H.R.; Anthonsen, T. Stable, highly unsaturated glyceridesEnzymatic synthesis with a carotenoic acid. Angew. Chem. Int. Ed. 1996, 35, 329-330.

43. Broszeit, G.; Diepenbrock, F.; Graf, O.; Hecht, D.; Heinze, J.; Martin, H.D.; Mayer, B.; Schaper, K.; Smie, A.; Strehblow, H.H. Vinylogous $\beta$-carotenes: Generation, storage, and delocalization of charge in carotenoids. Liebigs Ann. 1997, 1997, 2205-2213. 
44. Larsen, E.; Abendroth, J.; Partali, V.; Schulz, B.; Sliwka, H.R.; Quartey, E.G.K. Combination of vitamin $\mathrm{E}$ with a carotenoid: $\alpha$-tocopherol and trolox linked to $\beta$-apo-8'-carotenoic acid. Chem. Eur. J. 1998, 4, 113-117.

45. Naalsund, T.; Malterud, K.E.; Partali, V.; Sliwka, H.R. Synthesis of a triantioxidant compound: Combination of beta-apo-8'-carotenoic acid, selenacapryloic acid and trolox in a triglyceride. Chem. Phys. Lipids 2001, 112, 59-65.

46. Levy, L.W.; Binnington, R.H.; Tabatznik, A.S. Novel carotenoid ester. U.S. Patent 0,169,334, 14 November 2002.

47. Jørgensen, M.; Krebs, F.C. Stepwise and directional synthesis of end-functionalized singleoligomer OPVs and their application in organic solar cells. J. Org. Chem. 2004, 69, 6688-6696.

48. Beutner, S.; Frixel, S.; Ernst, H.; Hoffmann, T.; Hernandez-Blanco, I.; Hundsdörfer, C.; Kiesendahl, N.; Kock, S.; Martin, H.D.; Mayer, B.; et al. Carotenylflavonoids, a novel group of potent, dual-functional antioxidants. ARKIVOC 2007, VIII, 279-295.

49. Foss, B.J.; Nalum Naess, S.; Sliwka, H.R.; Partali, V. Stable and highly water-dispersible, highly unsaturated carotenoid phospholipids - Surface properties and aggregate size. Angew. Chem. Int. Ed. 2003, 42, 5237-5240.

50. Sliwka, H.R.; Partali, V.; Lockwood, S.F. Hydrophilic Carotenoids: Carotenoid Aggregates. In Carotenoids: Physical, Chemical, and Biological Functions and Properties; Landrum, J.T., Ed.; CRC Press: Boca Raton, FL, USA, 2009; pp. 31-58.

51. Øpstad, C.L.; Sliwka, H.R.; Partali, V. New colours for carotenoids-Synthesis of pyran polyenes. Eur. J. Org. Chem. 2010, 2010, 435-439.

52. Johansen, J.E.; Liaaen-Jensen, S. Algal Carotenoids 13. Chemical-reactions of allenic carotenoids. Acta Chem. Scand. 1974, B 28, 949-954.

53. Weedon, B.C.L.; Moss, G.P. Structure and nomenclature. In Carotenoids; Britton, G., Liaaen-Jensen, S., Pfander, H., Eds.; Birkhäuser: Basel, Switzerland, 1995; Volume 1A, p. 57.

54. Kuhn, R. Xanthophylle. Z. Physiol. Chem. 1931, 197, 141. 


\section{List of Xenobiotic Carotenoids}

\subsection{Halogen-Carotenoids}

6.1.1. Fluorine

F

1F 9-trifluoromethyl- $\beta, \beta$-carotene

$\mathrm{C}_{40} \mathrm{H}_{53} \mathrm{~F}_{3}$<smiles>CC1=C(/C=C/C(C)=C/C=C/C(C)=C/C=C/C=C(C)/C=C/C=C(\C=C\C2=C(C)CCCC2(C)C)C(F)(F)F)C(C)(C)CCC1</smiles>

D. Hoischen, L.U. Colmenares, I. Koukhareva, M. Ho, R.S.H. Liu, J. Fluorine Chem. 1999, 97, 165

2F 13-trifluoromethyl- $\beta, \beta$-carotene

$\mathrm{C}_{40} \mathrm{H}_{53} \mathrm{~F}_{3}$<smiles>C=C1CCC(C)(C)C(/C=C/C(C)=C/C=C/C(C)=C/C=C/C=C(/C=C/C=C(C)/C=C/C2=C(C)CCCC2(C)C)C(F)(F)F)=C1C</smiles>

D. Hoischen, L.U. Colmenares, I. Koukhareva, M. Ho, R.S.H. Liu, J. Fluorine Chem. 1999, 97, 165

3F $\quad 9,9$ '-bis(trifluoromethyl)- $\beta$-carotene<smiles>CC(=C/C=C/C(C)=C/C=C/C=C(C)/C=C/C=C(/C=C/C1=C(C)CCCC1(C)C)C(F)(F)F)C1(C)CCCCC1(C)C</smiles>

$\mathrm{C}_{40} \mathrm{H}_{50} \mathrm{~F}_{6}$

D. Hoischen, L.U. Colmenares, I. Koukhareva, M. Ho, R.S.H. Liu, J. Fluorine Chem. 1999, 97, 165

4F $\quad 13,13$ '-bis(trifluoromethyl)- $\beta$-carotene<smiles>CC1=C(/C=C/C(C)=C\C=C\C(=C/C=C/C=C(/C=C/C=C(C)/C=C/C2=C(C)CCCC2(C)C)C(F)(F)F)C(F)(F)F)C(C)(C)CCC1</smiles>

$\mathrm{C}_{40} \mathrm{H}_{50} \mathrm{~F}_{6}$

D. Hoischen, L.U. Colmenares, I. Koukhareva, M. Ho, R.S.H. Liu, J. Fluorine Chem. 1999, 97, 165 
5F 9-trifluoromethyl echinenone

$\mathrm{C}_{40} \mathrm{H}_{51} \mathrm{~F}_{3} \mathrm{O}$<smiles>CC1=C(/C=C/C(=C\C=C\C(C)=C\C=C\C=C(C)\C=C\C=C(C)\C=C\C2=C(C)C(=O)CCC2(C)C)C(F)(F)F)C(C)(C)CCC1</smiles>

D. Hoischen, L.U. Colmenares, I. Koukhareva, M. Ho, R.S.H. Liu, J. Fluorine Chem. 1999, 97, 165

6F 13-trifluoromethyl echinenone

$\mathrm{C}_{40} \mathrm{H}_{51} \mathrm{~F}_{3} \mathrm{O}$<smiles>CC1=C(/C=C/C(C)=C/C=C/C(C)=C/C=C/C=C(C)/C=C/C=C(C)/C=C/C2=C(C)C(=O)CCC2(C)C)C(C)(C)CCC1</smiles>

D. Hoischen, L.U. Colmenares, I. Koukhareva, M. Ho, R.S.H. Liu, J. Fluorine Chem. 1999, 97, 165

7F 9-trifluoromethyl canthaxanthin

$\mathrm{C}_{40} \mathrm{H}_{49} \mathrm{~F}_{3} \mathrm{O}_{2}$<smiles>CC1=C(/C=C/C(C)=C/C=C/C(C)=C/C=C/C=C(C)/C=C/C=C(\C)C(F)(F)F)C(C)(C)CCC1=O</smiles>

D. Hoischen, L.U. Colmenares, I. Koukhareva, M. Ho, R.S.H. Liu, J. Fluorine Chem. 1999, 97, 165

8F 13-trifluoromethyl canthaxanthin

$\mathrm{C}_{40} \mathrm{H}_{49} \mathrm{~F}_{3} \mathrm{O}_{2}$<smiles>CC1=C(/C=C/C(C)=C/C=C/C(C)=C/C=C/C=C(C)/C=C/C=C(C)/C=C/C2=C(C)C(=O)CCC2(C)C)C(C)(C)CCC1=O</smiles>

D. Hoischen, L.U. Colmenares, I. Koukhareva, M. Ho, R.S.H. Liu, J. Fluorine Chem. 1999, 97, 165 


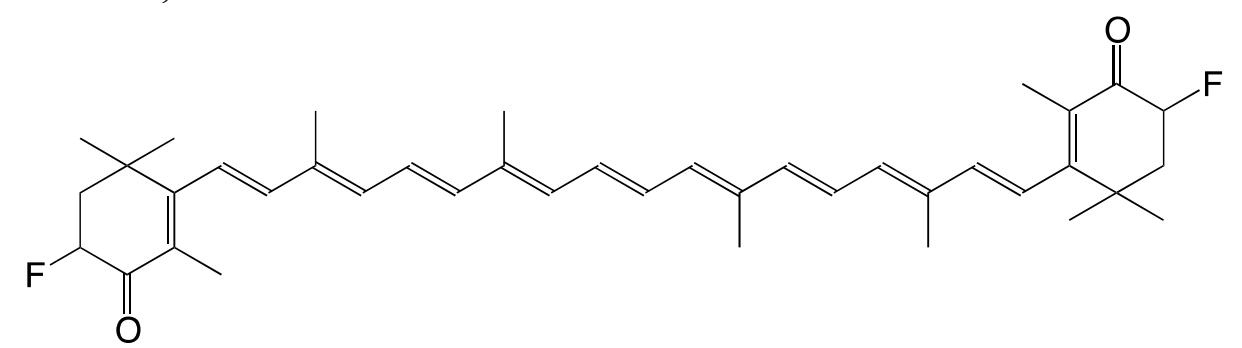

R.S.H. Liu, J. Liu, J. Nat. Prod. 2011, 74, 512

10F 10-fluoro-astaxanthin

$\mathrm{C}_{40} \mathrm{H}_{51} \mathrm{FO}_{4}$<smiles>CC1=C(/C=C/C(C)=C(F)/C=C/C(C)=C/C=C/C=C(C)/C=C/C=C(C)/C=C/C2=C(C)C(=O)C(O)CC2(C)C)C(C)(C)CC(O)C1=O</smiles>

R.S.H. Liu, J. Liu, J. Nat. Prod. 2011, 74, 512

11F 14-fluoro-astaxanthin

$\mathrm{C}_{40} \mathrm{H}_{51} \mathrm{FO}_{4}$<smiles>CC1=C(/C=C/C(C)=C/C=C/C(C)=C(F)/C=C/C=C(C)/C=C/C=C(C)/C=C/C2=C(C)C(=O)C(O)CC2(C)C)C(C)(C)CC(O)C1=O</smiles>

R.S.H. Liu, J. Liu, J. Nat. Prod. 2011, 74, 512

12F 3,3',10,10',14-pentafluoro-canthaxanthin

$\mathrm{C}_{40} \mathrm{H}_{47} \mathrm{~F}_{5} \mathrm{O}_{2}$<smiles>CC1=C(/C=C/C(C)=C(F)/C=C/C=C(C)/C=C/C(C)=C(F)/C=C/C(F)=C(C)/C=C/C2=C(C)C(=O)C(F)CC2(C)C)C(C)(C)CC(F)C1=O</smiles>

R.S.H. Liu, J. Liu, J. Nat. Prod. 2011, 74,512 
$\phi$-carotenoids

13F $\quad 1^{\prime}, 2^{\prime}, 3^{\prime}, 4^{\prime}, 5^{\prime}$-pentafluoro- $\beta, \phi$-carotene

$\mathrm{C}_{37} \mathrm{H}_{41} \mathrm{~F}_{5}$<smiles>CC1=C(/C=C/C(C)=C/C=C/C=C(C)/C=C/C=C/C=C(C)/C=C/c2c(F)c(F)c(F)c(F)c2F)C(C)(C)CCC1</smiles>

E. Hand, K.A. Belmore, L.D. Kispert, Helv. Chim. Acta 1993, 76, 1928

\subsubsection{Chlorine}

\section{Cl}

$\mathrm{Cl}$ crocetin dichloride

$\mathrm{C}_{20} \mathrm{H}_{22} \mathrm{Cl}_{2} \mathrm{O}_{2}$<smiles>CC(/C=C/C=C(\C)C(=O)Cl)=C\C=C\C=C(/C)C(=O)Cl</smiles>

H. Pfander, F. Wittwer, Helv. Chim. Acta 1979, 62, 1944

$2 \mathrm{Cl}$ norbixin dichloride<smiles>CC(/C=C/C=C(C)/C=C/C(=O)Cl)=C\C=C\C=C(C)\C=C\C=C(C)\C=C\C(=O)Cl</smiles>

$\mathrm{C}_{24} \mathrm{H}_{26} \mathrm{Cl}_{2} \mathrm{O}_{2}$

L. Levy, R.H. Binnington, A. Tabatnik, WO 02/068385, 2002

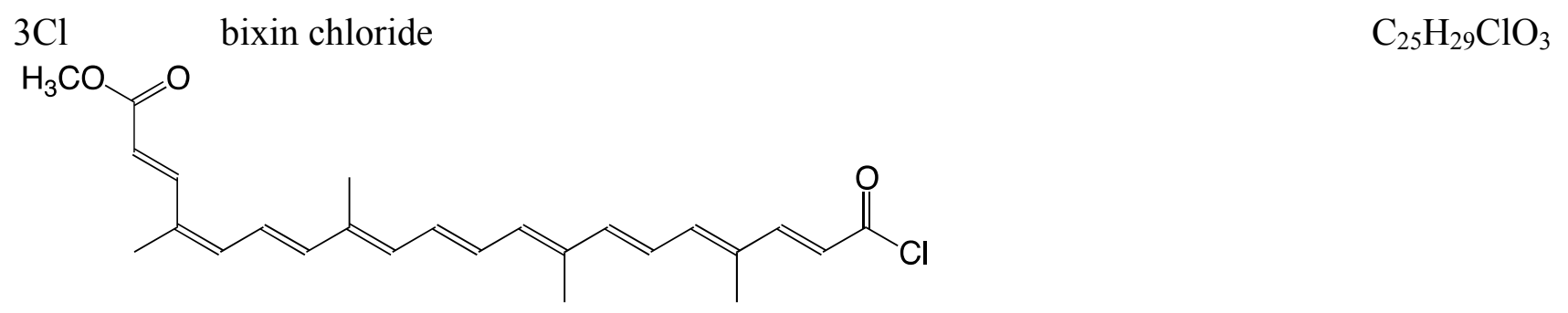

G. Ferrari, V. Vecchietti, EP 30009, 1981

T. Komatsu, E. Tsuchia, C. Böttcher, D. Donner, C. Messerschmidt, U. Siggel, W. Stocker, J.P. Rabe, J.H. Fuhrhop, J. Am. Chem. Soc. 1997, 119, 11660 
4Cl $\beta$-apo-8'-carotenoyl chloride, C30-acid chloride<smiles>CC1=C(/C=C/C(C)=C/C=C/C(C)=C/C=C/C=C(C)/C=C/C=C(\C)C(=O)Cl)C(C)(C)CCC1</smiles>

T. Naalsund, K.E. Malterrud, V. Partali, H.R. Sliwka, Chem. Phys. Lipids 2001, 112, 59

L. Levy, R. H. Binnington, A. Tabatnik, WO 02/068385, 2002

$5 \mathrm{Cl}$ 4-chloro- $\beta, \beta$-carotene

$\mathrm{C}_{40} \mathrm{H}_{55} \mathrm{Cl}$<smiles>CC1=C(/C=C/C(C)=C/C=C/C(C)=C/C=C/C=C(C)/C=C/C=C(C)/C=C/C2=C(C)C(Cl)CCC2(C)C)C(C)(C)CCC1</smiles>

H. Pfander, U. Leuenberger, Chimia 1976, 30, 71

6Cl 4-chloro-3',4'-didehydro- $\beta, \beta$-carotene<smiles>CC1=C(/C=C/C(C)=C/C=C/C(C)=C/C=C/C=C(C)/C=C/C=C(C)/C=C/C2C(C)C=CCC2(C)C)C(C)(C)CCC1Cl</smiles>

$\mathrm{C}_{40} \mathrm{H}_{53} \mathrm{Cl}$

H. Pfander, U. Leuenberger, Chimia 1976, 30, 71

$7 \mathrm{Cl} \quad 4$ '-chloro- $\beta, \beta$-caroten-3-ol

$\mathrm{C}_{40} \mathrm{H}_{55} \mathrm{ClO}$<smiles>CC1=C(/C=C/C(C)=C/C=C/C(C)=C/C=C/C(C)=C/C=C/C=C(C)/C=C/C2=C(C)C(Cl)CCC2(C)C)C(C)(C)CC(O)C1</smiles>

H. Pfander, U. Leuenberger, Chimia 1976, 30, 71 
$8 \mathrm{Cl} 4,4$ '-dichloro- $\beta, \beta$-carotene

$\mathrm{C}_{40} \mathrm{H}_{54} \mathrm{Cl}_{2}$<smiles>CC1=C(/C=C/C(C)=C/C=C/C(C)=C/C=C/C=C(C)/C=C/C=C(C)/C=C/C2=C(C)C(Cl)CCC2(C)C)C(C)(C)CCC1Cl</smiles>

H. Pfander, U. Leuenberger, Chimia 1976, 30, 71

9Cl 5,5'-dichloro-4,5,4',5'-tetrahydroisocarotene

$\mathrm{C}_{41} \mathrm{H}_{58} \mathrm{Cl}_{2}$<smiles>CC(/C=C/C=C(C)/C=C/C=C(C)/C=C/C=C(C)/C=C/C=C1C(C)(C)CCCC1(C)C)=C\C=C1C(C)(C)CC(C)CC1(C)Cl</smiles>

C. Bodea, E. Nicoara, Acad. rep. populare Romîne, Filiala Cluj, Studii Cercetâri Chim. 1959, 10, 1959

$10 \mathrm{Cl}$ 7-chloro-mutatoxanthin-3,3'-diacetate

$\mathrm{C}_{44} \mathrm{H}_{59} \mathrm{ClO}_{5}$<smiles>CC(=O)OC1CC(C)=C(/C(Cl)=C/C(C)=C/C=C/C(C)=C/C=C/C=C(C)/C=C/C=C(\C)C2C=C3C(C)(C)CC(OC(C)=O)CC3(C)O2)C(C)(C)C1</smiles>

J.E. Johansen, S. Liaaen-Jensen, Acta Chem. Scand. 1974, B28, 949

R. Buchecker, S. Liaaen-Jensen, Helv. Chim. Acta 1975, 58, 89

$11 \mathrm{Cl}$ 3'-chloro-4,5-dehydro-5-dehydroxy-neochrome

$\mathrm{C}_{37} \mathrm{H}_{49} \mathrm{ClO}_{2}$<smiles>CC(C=CC=C=C1C(C)=CC(O)CC1(C)C)=CC=CC=C(C)C=CC=C(C)C1C=C2C(C)(C)CC(Cl)CC2(C)O1</smiles>

R. Buchecker, S. Liaaen-Jensen, Helv. Chim. Acta 1975, 58, 89 
12Cl 3'-chloro-2,3-didehydro-5,18-dehydro-5-dehydroxy-neochrome<smiles>C=C1CC=CC(C)(C)C1=C=CC=CC(C)=CC=CC=C(C)C=CC=C(C)C1C=C2C(C)(C)CC(Cl)CC2(C)O1</smiles>

R. Buchecker, S. Liaaen-Jensen, Helv. Chim. Acta 1975, 58, 89

13Cl 7-chloro-mutatoxanthin-19',11'-olide 3-acetate<smiles>CC(=O)OC1CC(C)=C(/C(Cl)=C/C(C)=C/C=C/C(C)=C/C=C/C=C(C)/C=C2/C=C(C3C=C4C(C)(C)CC(O)CC4(C)O3)C(=O)O2)C(C)(C)C1</smiles>

J.E. Johansen, S. Liaaen-Jensen, Acta Chem. Scand. 1974, B28, 949

$14 \mathrm{Cl}$ 3'-chloro-6,7-didehydro-peridinol-3-acetate

$\mathrm{C}_{42} \mathrm{H}_{53} \mathrm{ClO}_{6}$<smiles></smiles>

J.E. Johansen, S. Liaaen-Jensen, Acta Chem. Scand. 1974, B28, 949

$15 \mathrm{Cl} \quad 4,5$-didehydro-5-dehydroxy-3'-chloro-peridinin-3-acetate

$\mathrm{C}_{42} \mathrm{H}_{51} \mathrm{ClO}_{5}$<smiles>CC(=O)OC1C=C(C)C(=C=C(C)C=CC=C(C)C=CC=CC=C(C)C=C2C=C(C=CC34OC3(C)CC(Cl)CC4(C)C)C(=O)O2)C(C)(C)C1</smiles>

J.E. Johansen, S. Liaaen-Jensen, Acta Chem. Scand. 1974, B28, 949 
$1 \mathrm{Br} \quad$ 20-bromo-crocetindial

$\mathrm{C}_{20} \mathrm{H}_{23} \mathrm{BrO}_{2}$<smiles>CC(C=O)=CC=CC(C)=CC=CC=C(C=CC=C(C)C=O)CBr</smiles>

J.E. Johansen, S. Liaaen-Jensen, Acta Chem. Scand. 1975, B29, 315

2Br 20,20'-dibromo-crocetindial

$\mathrm{C}_{20} \mathrm{H}_{22} \mathrm{Br}_{2} \mathrm{O}_{2}$<smiles>CC(C=O)=CC=CC(=CC=CC=C(CBr)CBr)CBr</smiles>

J.E. Johansen, S. Liaaen-Jensen, Acta Chem. Scand. 1975, B29, 315

$3 \mathrm{Br} \quad 4$-bromo- $\beta, \beta$-carotene<smiles>CC1=C(/C=C/C(C)=C/C=C/C(C)=C/C=C/C=C(C)/C=C/C=C(C)/C=C/C2=C(C)C(Br)CCC2(C)C)C(C)(C)CCC1</smiles>

R. Entschel, P. Karrer, Helv. Chim. Acta 1958, 41, 983

J. Morel, DE2001957, 1970

$4 \mathrm{Br} \quad 4,4$ '-dibromo- $\beta, \beta$-carotene

$\mathrm{C}_{40} \mathrm{H}_{58} \mathrm{Br}_{2}$<smiles>CC1=C(/C=C/C(C)=C/C=C/C(C)=C/C=C/C=C(C)/C=C/C=C(C)/C=C/C2=C(C)C(Br)CCC2(C)C)C(C)(C)CCC1Br</smiles>

R. Entschel, P. Karrer, Helv. Chim. Acta 1958, 41, 402

C. Martin, P. Karrer, Helv. Chim. Acta 1959, 42, 464 
$5 \mathrm{Br} \quad 4$-bromo-4'-ethoxy- $\beta, \beta$-carotene

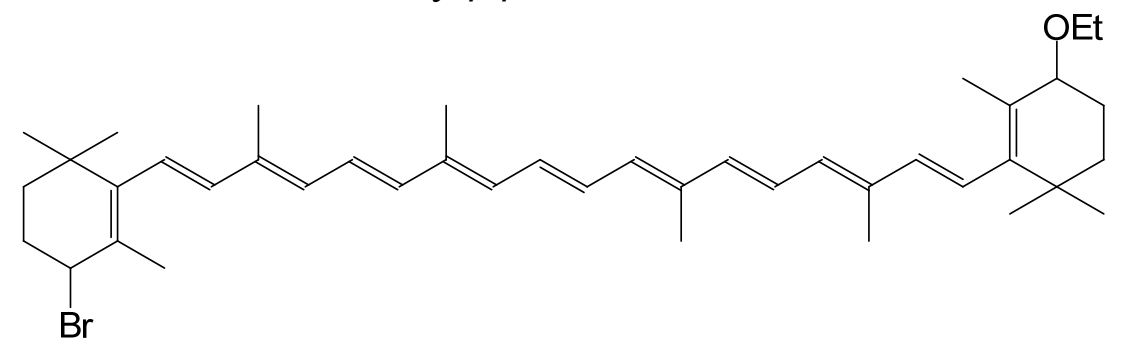

R. Entschel, P. Karrer, Helv. Chim. Acta 1958, 41, 402

$6 \mathrm{Br} \quad 4$-bromo-4'-ethoxy- $\beta, \beta$-carotene

$\mathrm{C}_{44} \mathrm{H}_{63} \mathrm{BrO}_{2}$

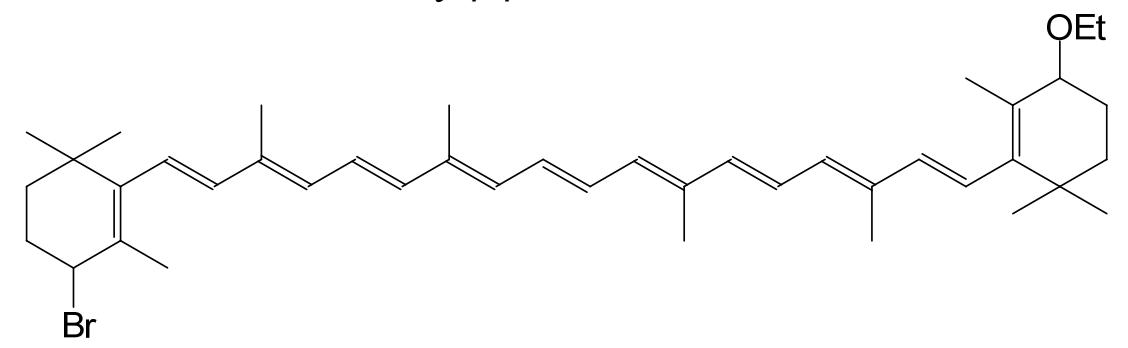

R. Entschel, P. Karrer, Helv. Chim. Acta 1958, 41, 402

7Br 4-bromo-4-ethoxy echinenone

$\mathrm{C}_{42} \mathrm{H}_{57} \mathrm{BrO}_{2}$

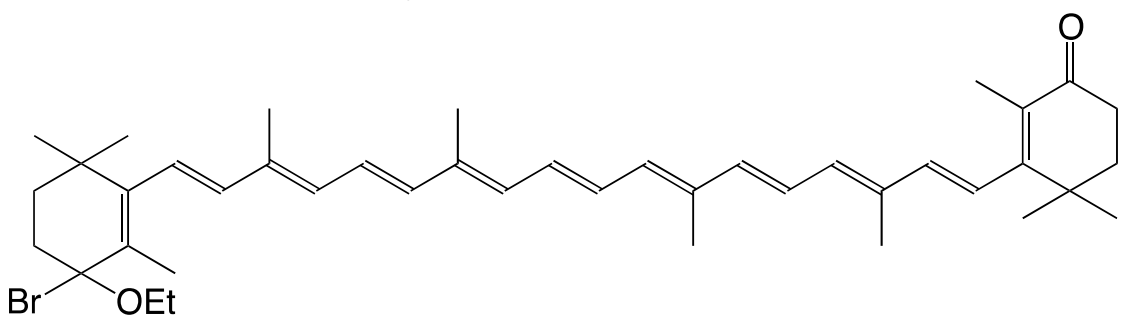

R. Entschel, P. Karrer, Helv. Chim. Acta 1958, 41, 402

$8 \mathrm{Br} \quad 4$ bromo-4',4'-diethoxy- $\beta, \beta$-carotene

$\mathrm{C}_{44} \mathrm{H}_{63} \mathrm{BrO}_{2}$

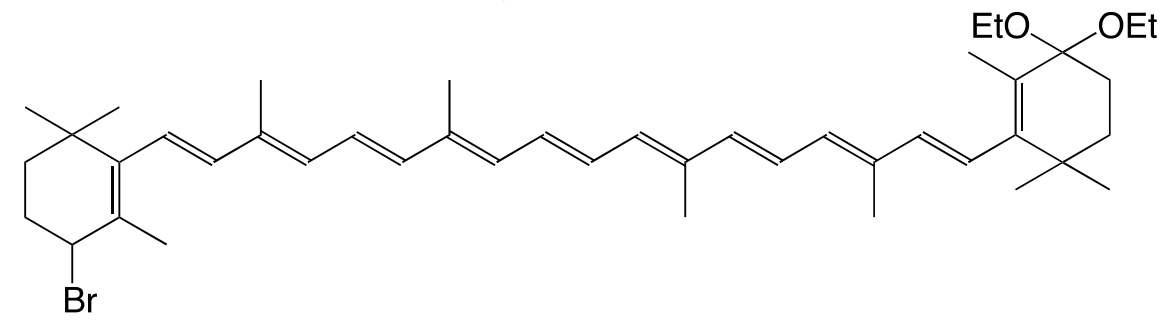

F.J. Petracek, L. Zechmeister, J. Am. Chem. Soc. 1956, 78, 1427 
$9 \mathrm{Br} \quad 4,4,4$ '-tribromo- $\beta, \beta$-carotene

$\mathrm{C}_{40} \mathrm{H}_{53} \mathrm{Br}_{3}$<smiles>CC1=C(/C=C/C(C)=C/C=C/C(C)=C/C=C/C=C(C)/C=C/C=C(C)/C=C/C2=C(C)C(Br)CCC2(C)C)C(C)(C)CCC1Br</smiles>

F.J. Petracek, L. Zechmeister, J. Am. Chem. Soc. 1956, 78, 1427

$10 \mathrm{Br}$ 7-bromo-mutatoxanthin-diacetate

$\mathrm{C}_{44} \mathrm{H}_{59} \mathrm{BrO}_{5}$<smiles>CC(=O)OC1CC(C)=C(/C(Br)=C/C(C)=C/C=C/C(C)=C/C=C/C=C(C)/C=C/C=C(\C)C2C=C3C(C)(C)CC(OC(C)=O)CC3(C)O2)C(C)(C)C1</smiles>

R. Buchecker, S. Liaaen-Jensen, Helv. Chim. Acta 1975, 58, 89

6.1.4. Iodine

$1 \mathrm{I} \quad 5,5^{\prime}$-diiodo-5,6,5',6'-didehydro- $\beta, \beta$-carotene

$\mathrm{C}_{40} \mathrm{H}_{58} \mathrm{I}_{2}$<smiles>CC(/C=C/C=C/C1C(C)(C)CCCC1(C)C)=C\C=C\C=C(C)\C=C\C=C(C)\C=C\C1C(C)(C)CCCC1(C)C</smiles>

unconfirmed structure

B.G. Savinov, G.S. Tretyakova, Vitaminy Akad. Nauk Ukr. S.S.R 1953, 1, 137

Other carotenoid-iodine compounds are formulated as ionic complexes:

B.F. Lutnaes, J. Krane, S. Liaaen-Jensen, Org. Biomol. Chem. 2004, 2, 2821 
1Si (= 2๑S) 7,5'-diapo-7-thienyl-carotene-5'-triethoxysilane $\mathrm{C}_{34} \mathrm{H}_{46} \mathrm{O}_{3} \mathrm{SSi}$<smiles>CCO[Si](/C=C/C=C/C(C)=C/C=C/C(C)=C/C=C/C=C(C)/C=C/C=C(C)/C=C/c1cccs1)(OCC)OCC</smiles>

F. Effenberger, M. Wezstein, Synthesis 2001, 1368

6.3. Nitrogen-Carotenoids

$\mathbf{N}$

'́apo- $\beta$-carotene-8'-nitrile

$\mathrm{C}_{30} \mathrm{H}_{39} \mathrm{~N}$

Z. He, D. Gosztola, Y. Deng, G. Gao, M.R. Wasielewski, L.D. Kispert, J. Phys. Chem. B 2000, 104, 6668

2N 10'-apo-5,6-seco- $\beta$-carotene-10'-nitrile

$\mathrm{C}_{27} \mathrm{H}_{35} \mathrm{NO}_{2}$<smiles>CC(=O)CCCC(C)(C)C(=O)/C=C/C(C)=C/C=C/C(C)=C/C=C/C=C(C)/C=C/C#N</smiles>

R. Kuhn, H. Brockmann, Chem. Ber. 1934, 67, 885

3N 6'-apo- $\beta$-carotene-6'-nitrile

$\mathrm{C}_{32} \mathrm{H}_{41} \mathrm{~N}$<smiles>CC1=C(/C=C/C(C)=C/C=C/C=C/C(C)=C/C=C/C(C)=C/C=C/C(C)=C/C=C/C#N)C(C)(C)CCC1</smiles>

Z. He, D. Gosztola, Y. Deng, G. Gao, M.R. Wasielewski, L.D. Kispert, J. Phys. Chem. B 2000, 104, 6668

S. Tretiak, V. Chernyak, S. Mukamel, J. Am. Chem. Soc. 1997, 119, 11408

S. Gilmour, S.R. Marder, B.G. Tiemann, L.T. Cheng, J. Chem. Soc. Chem. Commun. 1993, 432 


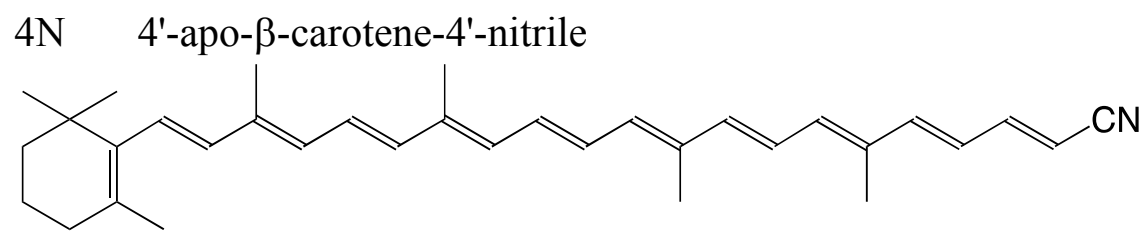

$\mathrm{C}_{34} \mathrm{H}_{43} \mathrm{~N}$

Z. He, D. Gosztola, Y. Deng, G. Gao, M.R. Wasielewski, L.D. Kispert, J. Phys. Chem. B 2000, 104, 6668

5N 7'-cyano- $\beta$-apo-7'-carotenoic acid methyl ester

$\mathrm{C}_{34} \mathrm{H}_{43} \mathrm{NO}_{2}$<smiles>COC(=O)/C(C#N)=C/C(C)=C/C=C/C(C)=C/C=C/C=C(C)/C=C/C=C(C)/C=C/C1=C(C)CCCC1(C)C</smiles>

H. Ikeda, T. Sakai, Y. Kawabe, JP 2-2534, 1990

$6 \mathrm{~N} \quad 7^{\prime}$-apo-7',7'-dicyano- $\beta$-carotene

$\mathrm{C}_{33} \mathrm{H}_{40} \mathrm{~N}_{2}$<smiles>C/C=C(C)/C=C/C=C(C)/C=C/C=C(C)/C=C/C=C/C=C(C)/C=C/C=C(C)/C=C(\C)C#N</smiles>

M. Blanchard-Desce, I. Ledoux, J.M. Lehn, J. Malthête, J. Zyss, J. Chem. Soc. Chem. Commun. 1988, 737

H. Ikeda, T. Sakai, Y. Kawabe, JP 2-2534, 1990

M.P. O’Neil, M.R. Nasielewski, M.M. Khaled, L.D. Kispert, J. Chem. Phys. B, 1991, 95, 7212

S. Gilmour, S.R. Marder, B.G. Tiemann, L.T. Cheng, J. Chem. Soc. Chem. Commun. 1993, 432

E.S. Hand, K.A. Belmore, L.D. Kispert, J. Chem. Soc. Perkin Trans 2, 1993, 659

S. Tretiak, V. Chernyak, S. Mukamel, J. Am. Chem. Soc. 1997, 119, 11408

A.J. Cruz, K. Siam, D.P. Rillema, J. Phys. Chem. 2011, 115, 1108

$7 \mathrm{~N} \quad 4,14$ '-dicyano-20,20'-dinor- $\beta, \beta$-carotene-

$\mathrm{C}_{40} \mathrm{H}_{50} \mathrm{~N}_{2}$<smiles>CC1=C(/C=C/C(C)=C/C=C/C=C(C#N)/C=C/C(C#N)=C/C=C/C=C(C)/C=C/C2=C(C)CCCC2(C)C)C(C)(C)CCC1</smiles>

H.H. Haeck, T. Kralt, Rec. Trav. Chim. Pays-Bas 1966, 85, 343

P.B. Braun, J. Hornstra, J.I. Leenhouts, Acta Cryst. 1971, B27, 90 
$8 \mathrm{~N}$ dicyano-C44:14

$\mathrm{C}_{48} \mathrm{H}_{60} \mathrm{~N}_{2}$<smiles>CC1=C(/C=C/C(C)=C/C=C/C(C)=C/C=C(C#N)/C=C/C(C#N)=C/C=C/C=C(C)/C=C/C=C(C)/C=C/C2=C(C)CCCC2(C)C)C(C)(C)CCC1</smiles>

H.H. Haeck, T. Kralt, Rec. Trav. Chim. Pays-Bas 1966, 85, 343

9N dicyano-C48:15<smiles>CC1=C(/C=C/C(C)=C/C=C/C(C)=C/C=C/C(C)=C(C#N)/C=C/C(C#N)=C(C)/C=C/C=C(C)/C=C/C=C(C)/C=C/C2=C(C)CCCC2(C)C)C(C)(C)CCC1</smiles>

H.H. Haeck, T. Kralt, Rec. Trav. Chim. Pays-Bas 1966, 85, 343

$10 \mathrm{~N} \quad(3 S)-2$ ',3'-didehydro- $\beta, \beta$-carotene-3-amine<smiles>CC1=C(/C=C/C(C)=C/C=C/C(C)=C/C=C/C=C(C)/C=C/C=C(C)/C=C/C2=C(C)C[C@@H](N)CC2(C)C)C(C)(C)C=CC1</smiles>

H.R. Sliwka, S. Liaaen-Jensen, Tetrahedron Asym. 1993, 4, 2377

$11 \mathrm{~N} \quad\left(3 R, 3^{\prime} S\right)-3$ '-amino- $\beta, \beta$-carotene-3-ol

$\mathrm{C}_{40} \mathrm{H}_{57} \mathrm{NO}$<smiles>CC1=C(/C=C/C(C)=C/C=C/C(C)=C/C=C/C=C(C)/C=C/C=C(C)/C=C/C2=C(C)C[C@H](O)CC2(C)C)C(C)(C)C[C@H](N)C1</smiles>

H.R. Sliwka, S. Liaaen-Jensen, Tetrahedron Asym. 1993, 4, 2377

$12 \mathrm{~N} \quad(3 S, 3$ 'S)- $\beta, \beta$-carotene-3,3'-amine

$\mathrm{C}_{40} \mathrm{H}_{58} \mathrm{~N}_{2}$<smiles>CC1=C(/C=C/C(C)=C/C=C/C(C)=C/C=C/C=C(C)/C=C/C=C(C)/C=C/C2=C(C)C[C@@H](N)CC2(C)C)C(C)(C)C[C@H](N)C1</smiles>

H.R. Sliwka, S. Liaaen-Jensen, Tetrahedron Asym. 1993, 4, 2377

J. Inananga, M. Yamaguchi, Mem. Fac. Sci. Kyushi Univ. Ser. C, 1989, 17, 109 
$13 \mathrm{~N} \quad\left(3 S, 3^{\prime} S\right)-\beta, \beta$-carotene-3,3'-diacetamide

$\mathrm{C}_{44} \mathrm{H}_{62} \mathrm{~N}_{2} \mathrm{O}_{2}$<smiles>C#CC(=O)N[C@H]1CC(C)=C(/C=C/C(C)=C/C=C/C(C)=C/C=C/C=C(C)/C=C/C=C(C)/C=C/C2=C(C)C[C@@H](NC(=O)NC3CCCCC3)CC2(C)C)C(C)(C)C1</smiles>

J. Inananga, M. Yamaguchi, Mem. Fac. Sci. Kyushi Univ. Ser. C, 1989, 17, 109

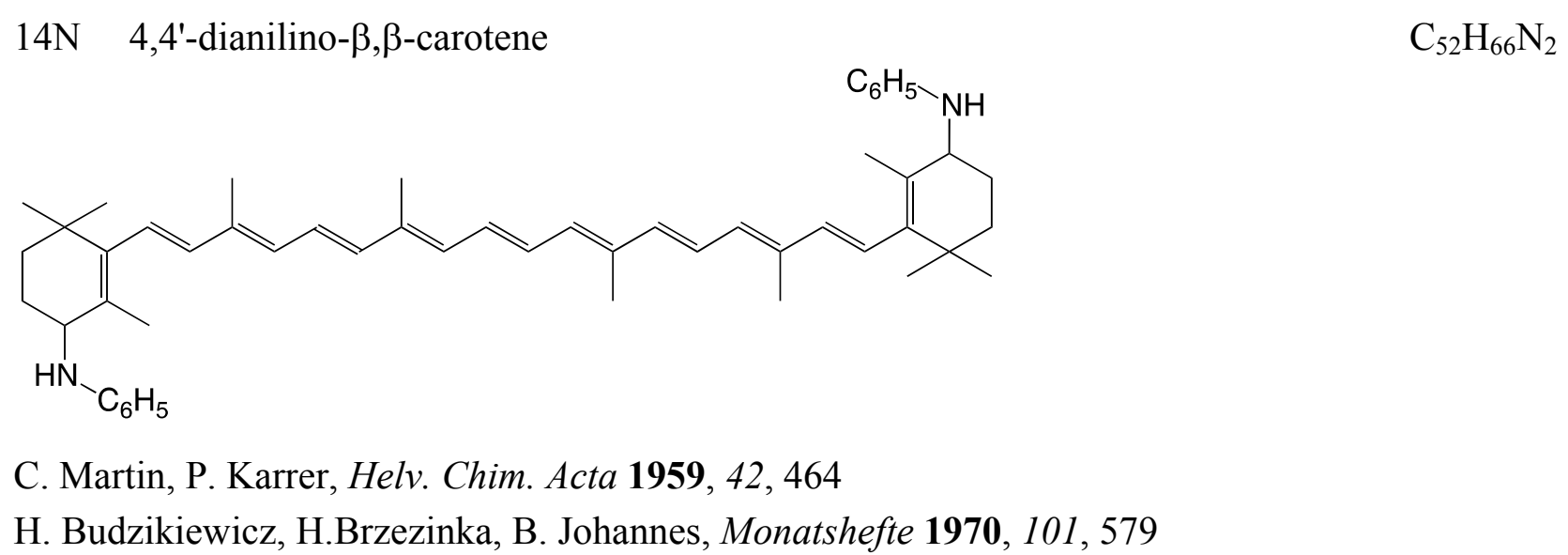

$15 \mathrm{~N} \quad 4,4$ '-bis $(N$-methyl-anilino)- $\beta, \beta$-carotene

$\mathrm{C}_{54} \mathrm{H}_{70} \mathrm{~N}_{2}$

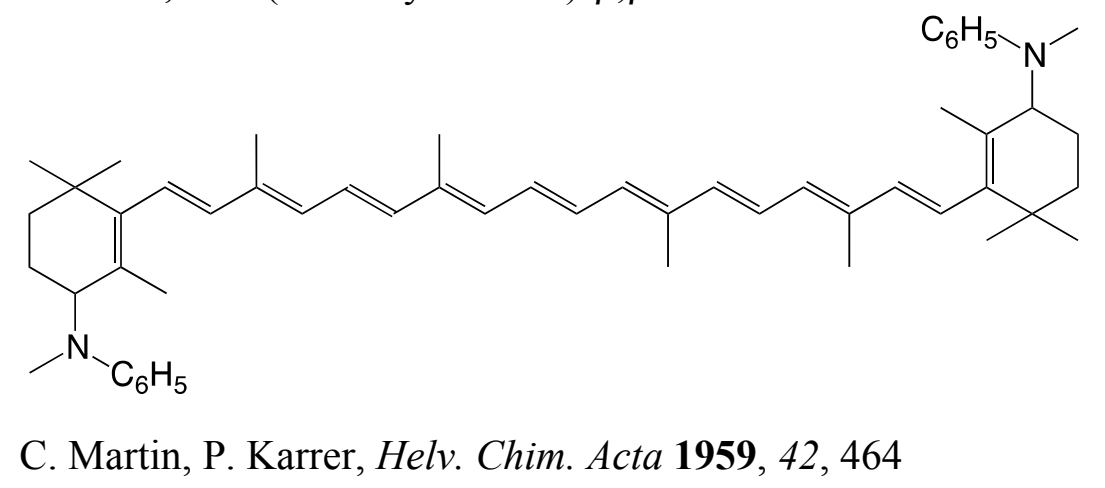

16N (3S)-3-azido-2',3'-didehydro- $\beta, \beta$-carotene

$\mathrm{C}_{40} \mathrm{H}_{53} \mathrm{~N}_{3}$<smiles>CC1=C(/C=C/C(C)=C/C=C/C(C)=C/C=C/C=C(C)/C=C/C=C(C)/C=C/C2=C(C)C[C@@H](N)CC2(C)C)C(C)(C)C=CC1</smiles>

H.R. Sliwka, S. Liaaen-Jensen, Tetrahedron Asym. 1993, 4, 2377

H.R. Sliwka, Helv. Chim. Acta 1999, 82, 161 
$17 \mathrm{~N} \quad(3 R, 3 ' S)-3$ '-azido- $\beta, \beta$-carotene-3-ol

$\mathrm{C}_{40} \mathrm{H}_{55} \mathrm{~N}_{3} \mathrm{O}$<smiles>CC1=C(/C=C/C(C)=C/C=C/C(C)=C/C=C/C=C(C)/C=C/C=C(C)/C=C/C2C(C)C[C@@H](N)CC2(C)C)C(C)(C)C[C@H](O)C1</smiles>

H.R. Sliwka, S. Liaaen-Jensen, Tetrahedron Asym. 1993, 4, 2377

H.R. Sliwka, Helv. Chim. Acta 1999, 82, 161

$18 \mathrm{~N} \quad(3 S, 3 ' S)$-diazido- $\beta, \beta$-carotene

$\mathrm{C}_{40} \mathrm{H}_{54} \mathrm{~N}_{6}$<smiles>CC1=C(/C=C/C(C)=C/C=C/C(C)=C/C=C/C=C(C)/C=C/C=C(C)/C=C/C2=C(C)C[C@@H](N)CC2(C)C)C(C)(C)CC(N)C1</smiles>

H.R. Sliwka, S. Liaaen-Jensen, Tetrahedron Asym. 1993, 4, 2377

J. Inananga, M. Yamaguchi, Mem. Fac. Sci. Kyushi Univ. Ser. C, 1989, 17, 109

H.R. Sliwka, Helv. Chim. Acta 1999, 82, 161

19N 8'-apo- $\beta$-caroten- 8 '-aldoxime hydrochloride, C30-aldoxime hydrochloride<smiles>CC1=C(/C=C/C(C)=C/C=C/C(C)=C/C=C/C=C(C)/C=C/C=C(C)/C=[NH+]\[O-])C(C)(C)CCC1</smiles>

J. Willibald, S. Rennebaum, S. Breukers, S.H. Abdel Hafez, A. Patel, C.L. Øpstad, R. Schmid, S. Nalum Naess, H.R. Sliwka, V. Partali, Chem. Phys. Lipids 2009, 161, 32

H.R. Sliwka, V. Partali, S.F. Lockwood, in Carotenoids, ed. J.T. Landrum, CRC Press, Boca Raton, USA, 2010, chpt. 3

20N echinenenon oxime hydrochloride<smiles>CC1=C(/C=C/C(C)=C/C=C/C(C)=C/C=C/C=C(C)/C=C/C=C(C)/C=C/C2=C(C)CCCC2(C)C)C(C)(C)CCC1</smiles>
$\mathrm{C}_{40} \mathrm{H}_{56} \mathrm{ClNO}$<smiles>CNCO</smiles>

J. Willibald, S. Rennebaum, S. Breukers, S.H. Abdel Hafez, A. Patel, C.L. Øpstad, R. Schmid, S. Nalum Naess, H.R. Sliwka, V. Partali, Chem. Phys. Lipids 2009, 161, 32 H.R. Sliwka, V. Partali, S.F. Lockwood, in Carotenoids, ed. J.T. Landrum, CRC Press, Boca Raton, USA, 2010, chpt. 3 
$21 \mathrm{~N}$ canthaxanthin dioxime hydrochloride

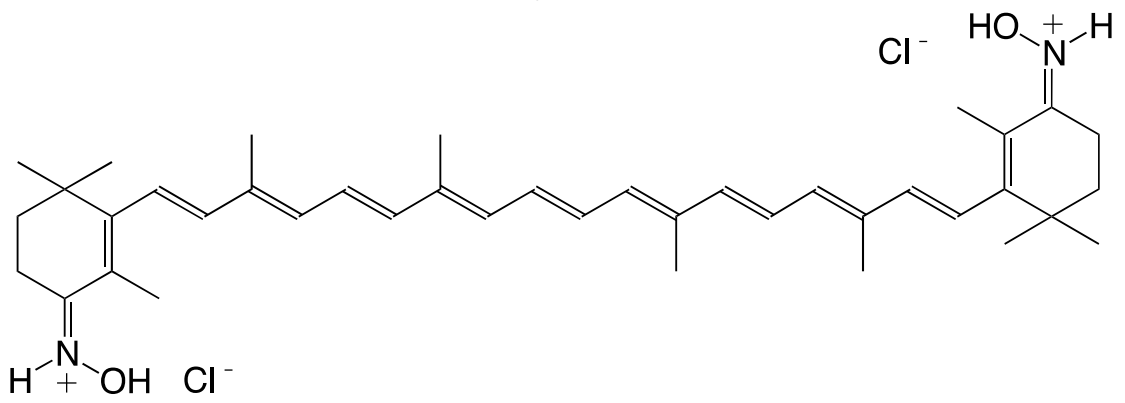

$\mathrm{C}_{40} \mathrm{H}_{56} \mathrm{Cl}_{2} \mathrm{~N}_{2} \mathrm{O}_{2}$

J. Willibald, S. Rennebaum, S. Breukers, S.H. Abdel Hafez, A. Patel, C.L. Øpstad, R. Schmid, S. Nalum Naess, H.R. Sliwka, V. Partali, Chem. Phys. Lipids 2009, 161, 32

H.R. Sliwka, V. Partali, S.F. Lockwood, in Carotenoids, ed. J.T. Landrum, CRC Press, Boca Raton, USA, 2010, chpt. 3

$22 \mathrm{~N}$ astaxanthin dioxime hydrochloride

$\mathrm{C}_{40} \mathrm{H}_{56} \mathrm{Cl}_{2} \mathrm{~N}_{2} \mathrm{O}_{4}$<smiles></smiles>

J. Willibald, S. Rennebaum, S. Breukers, S.H. Abdel Hafez, A. Patel, C.L. Øpstad, R. Schmid, S. Nalum Naess, H.R. Sliwka, V. Partali, Chem. Phys. Lipids 2009, 161, 32

H.R. Sliwka, V. Partali, S.F. Lockwood, in Carotenoids, ed. J.T. Landrum, CRC Press, Boca Raton, USA, 2010, chpt. 3

23N 7'-aza-7'-methyl-7'-apo- $\beta$-carotene

$\mathrm{C}_{31} \mathrm{H}_{43} \mathrm{~N}$<smiles>CN=C/C(C)=C/C=C/C(C)=C/C=C/C=C(C)/C=C/C=C(C)/C=C/C1=C(C)CCCC1(C)C</smiles>

G.A.J. Pitt, F.D. Collins, R.A. Morton, P. Stok, Biochem. J. 1955, 59, 122 
24N 7'-aza-7'-butyl-7'-apo- $\beta$-carotene

$\mathrm{C}_{34} \mathrm{H}_{49} \mathrm{~N}$<smiles>CCCCN=C/C(C)=C/C=C/C(C)=C/C=C/C=C(C)/C=C/C=C(C)/C=C/C1=C(C)CCCC1(C)C</smiles>

T.A. Moore, P.S. Song, J. Mol. Spec. 1974, 52, 224

$25 \mathrm{~N} \quad 7$-aza-7'-butyl-7'-apo- $\beta$-carotene hydrochloride

$\mathrm{C}_{35} \mathrm{H}_{52} \mathrm{ClN}$<smiles>CCCC[NH+]=C/C(C)=C/C=C/C(C)=C/C=C/C=C(C)/C=C/C=C(C)/C=C/C1=C(C)CCCC1(C)C</smiles>

T.A. Moore, P.S. Song, J. Mol. Spec. 1974, 52, 224

$26 \mathrm{~N} \quad 5$ '-aza-5'-butyl-5'-apo- $\beta$-carotene

$\mathrm{C}_{37} \mathrm{H}_{53} \mathrm{~N}$<smiles>CCCCN=C/C=C/C(C)=C/C=C/C(C)=C/C=C/C=C(C)/C=C/C=C(C)/C=C/C1=C(C)CCCC1(C)C</smiles>

T.A. Moore, P.S. Song, J. Mol. Spec. 1974, 52, 224

27N 5'-aza-5'-butyl-5'-apo- $\beta$-carotene hydrochloride<smiles>CCCC[NH+]=C/C=C/C(C)=C/C=C/C(C)=C/C=C/C=C(C)/C=C/C=C(C)/C=C/C1=C(C)CCCC1(C)C</smiles>

T.A. Moore, P.S. Song, J. Mol. Spec. 1974, 52, 224

28N 5'-aza-5'-butyl-5'-apo- $\beta$-caroten-3-ol

$\mathrm{C}_{36} \mathrm{H}_{51} \mathrm{NO}$<smiles>CCCCN=C/C=C/C(C)=C/C=C/C(C)=C/C=C/C=C(C)/C=C/C=C(C)/C=C/C1=C(C)CC(O)CC1(C)C</smiles>

T.A. Moore, P.S. Song, J. Mol. Spec. 1974, 52, 224 
29N 5'-aza-5'-butyl-5'-apo- $\beta$-caroten-3-ol hydrochloride

$\mathrm{C}_{36} \mathrm{H}_{52} \mathrm{ClNO}$<smiles>CCCC[NH+](Cl)/C=C/C=C(C)/C=C/C=C(C)/C=C/C=C/C(C)=C/C=C(C)/C=C/C1=C(C)CC(O)CC1(C)C</smiles>

T.A. Moore, P.S. Song, J. Mol. Spec. 1974, 52, 224

30N 7'-aza-7'-ureido-7'-apo- $\beta$-caroten-3-ol, $\beta$-citraurin semicarbazone<smiles>CC1=C(/C=C/C(C)=C/C=C/C(C)=C/C=C/C=C(C)/C=C/C=C(C)/C=N/NC(N)=O)C(C)(C)CC(O)C1</smiles>

L. Zechmeister, L. von Cholnoky, Liebigs Ann. 1937, 530, 291

31N 7'-nitro-7'-apo- $\beta$-carotene

$\mathrm{C}_{31} \mathrm{H}_{41} \mathrm{NO}_{2}$<smiles>CC1=C(/C=C/C(C)=C/C=C/C(C)=C/C=C/C=C(C)/C=C/C=C(\C)C=[N+]([O-])[O-])C(C)(C)CCC1</smiles>

H. Ikeda, T. Sakai, Y. Kawabe, JP 2-2534, 1990

$32 \mathrm{~N} \quad 4$-nitro- $\beta, \beta$-carotene<smiles>CC1=C(/C=C/C(C)=C/C=C/C(C)=C/C=C/C=C(C)/C=C/C=C(C)/C=C/C2=C(C)C([N+](=O)[O-])CCC2(C)C)C(C)(C)CCC1</smiles>

$\mathrm{C}_{40} \mathrm{H}_{55} \mathrm{NO}_{2}$

D.L. Baker, E.S. Kroll, N. Jacobsen, D.C. Liebler, Chem. Res. Toxicol. 1999, 12, 535 
33N 11-nitroastaxanthin

$\mathrm{C}_{40} \mathrm{H}_{51} \mathrm{NO}_{6}$<smiles>CC1=C(C=C/C(C)=C/C(C)=C/C=C/C=C(C)/C=C/C=C(C)/C=C/C2=C(C)C(=O)C(O)CC2(C)C)C(C)(C)CC(O)C1=O</smiles>

R. Yoshioka, T. Hayakawa, K. Ishuzuka, A. Kulkarni, Y. Terada, T. Maoka, H. Etoh, Tetrahedron Lett. 2006, 47, 3637 (cis-isomer)

34N 15-nitroastaxanthin

$\mathrm{C}_{40} \mathrm{H}_{51} \mathrm{NO}_{6}$<smiles>CC1=C(/C=C/C(C)=C/C=C/C(C)=C\C(=C/C=C(C)/C=C/C=C(C)/C=C/C2=C(C)C(=O)C(O)CC2(C)C)[N+](=O)[O-])C(C)(C)CC(O)C1=O</smiles>

R. Yoshioka, T. Hayakawa, K. Ishuzuka, A. Kulkarni, Y. Terada, T. Maoka, H. Etoh, Tetrahedron Lett. 2006, 47, 3637 (cis-isomer)

$35 \mathrm{~N} \quad 12-n i t r o c a p s a n t h i n$

$\mathrm{C}_{40} \mathrm{H}_{55} \mathrm{NO}_{5}$<smiles>CC1=C(/C=C/C(C)=C/C=C(C)/C=C/C=C(C)/C=C/C=C(C)/C(C)=C/C=C/C(=O)[C@@]2(C)C[C@@H](O)CC2(C)C)C(C)(C)C[C@H](O)C1</smiles>

M. Tsuboi, H. Etoh, K. Kato, H. Nakatugawa, H. Kato, Y. Maejima, G. Matsumoto, H. Mori, M. Hosokawa, K. Miyashita, H. Tokuda, N. Suzui, T. Maoka, J. Agric. Food Chem. 2011, 59, 10572

36N 11-nitrofucoxanthin

$\mathrm{C}_{42} \mathrm{H}_{57} \mathrm{NO}_{8}$<smiles>CCO[C@H]1CC(C)(C)C(=C=CC(C)=CC=CC(C)=CC=CC=C(C)C=C(C=C(C)C(=O)C[C@@]23O[C@@]2(C)C[C@@H](O)CC3(C)C)[N+](=O)[O-])[C@@](C)(O)C1</smiles>

M. Tsuboi, H. Etoh, K. Kato, H. Nakatugawa, H. Kato, Y. Maejima, G. Matsumoto, H. Mori, M. Hosokawa, K. Miyashita, H. Tokuda, N. Suzui, T. Maoka, J. Agric. Food Chem. 2011, 59, 10572 
37N 15-nitrofucoxanthin

$\mathrm{C}_{42} \mathrm{H}_{57} \mathrm{NO}_{8}$<smiles>CC(=O)O[C@H]1CC(C)(C)C(=C=CC(C)=CC=CC(C)=CC=C(C=C(C)C=CC=C(C)C(=O)CC23O[C@]2(C)C[C@@H](O)CC3(C)C)[N+](=O)[O-])[C@@](C)(O)C1</smiles>

M. Tsuboi, H. Etoh, K. Kato, H. Nakatugawa, H. Kato, Y. Maejima, G. Matsumoto, H. Mori, M.

Hosokawa, K. Miyashita, H. Tokuda, N. Suzui, T. Maoka, J. Agric. Food Chem. 2011, 59, 10572

38N $\quad 16,16$ '-diaza- $\beta, \beta$-carotene (diretinyliden hydrazine)

$\mathrm{C}_{40} \mathrm{H}_{56} \mathrm{~N}_{2}$<smiles>CC1=C(/C=C/C(C)=C/C=C/C(C)=C/C=N/N=C/C=C(C)/C=C/C=C(C)/C=C/C2=C(C)CCCC2(C)C)C(C)(C)CCC1</smiles>

T. Miki, Y. Hara, JP 34-002118, 1959

T. Miki, Y. Hara, Chem. Pharm. Bull. 1962, 10, 922

39N 5-acetyl-2-nor- $\beta$-apo-7'-carotenoic acid amide

$\mathrm{C}_{32} \mathrm{H}_{41} \mathrm{NO}_{2}$

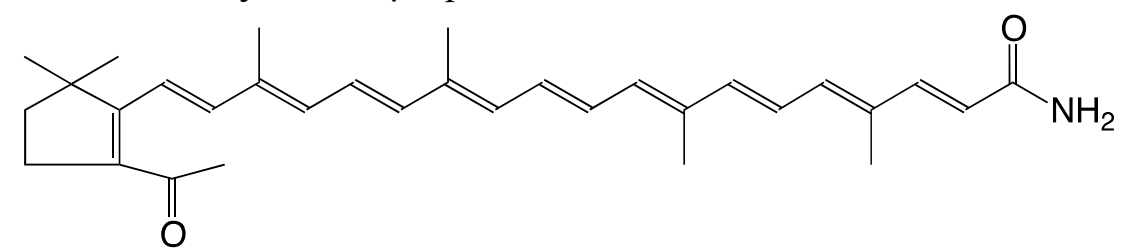

R. Kuhn, H. Brockmann, Chem. Ber. 1934, 67, 885

40N $\quad N$-hexyl crocetinamide

$\mathrm{C}_{26} \mathrm{H}_{35} \mathrm{NO}_{3}$<smiles>CC(/C=C/C=C(\C)C(=O)O)=C\C=C\C=C(C)\C=C\C=C(/C)C(=O)NC1CCCCC1</smiles>

G. Quinkert, K.R. Schmieder, G. Dürner, K. Hache, A. Stegk, D.H.R. Barton, Chem. Ber. 1977, 110, 3582 
41N $\beta$-apo-7'-benzoylamino-7'-carotenoic acid $N, N$-diethylamide $\mathrm{C}_{43} \mathrm{H}_{56} \mathrm{~N}_{2} \mathrm{O}_{2}$<smiles>CCCCCC(=O)N/C(=C\C(C)=C\C=C\C(C)=C\C=C\C=C(C)\C=C\C=C(C)\C=C\C1=C(C)CCCC1(C)C)C(=O)N(CC)CC</smiles>

M. Tomoaia-Cotisel, J. Zsako, E. Chifu, D.A. Ladenhead, Langmuir 1990, 6,191. The authors list several related amides.

$42 \mathrm{~N} \quad \beta$-apo-7'-benzoylamido-7'-carotenoic acid $N$-aminoethylamide

$\mathrm{C}_{41} \mathrm{H}_{53} \mathrm{~N}_{3} \mathrm{O}_{2}$<smiles>CCCCCCCCC(=O)NC(=CC(C)=CC=CC(C)=CC=CC=C(C)C=CC=C(C)C=CC1=C(C)CCCC1(C)C)C(=O)NCCN</smiles>

M. Tomoaia-Cotisel, J. Zsako, E. Chifu, D.A. Ladenhead, Langmuir 1990, 6,191

43N $\beta$-apo-7'-benzoylamido-7'-carotenoic acid $N$-methyl-N-(2-hydroxyethyl)-amide

$\mathrm{C}_{42} \mathrm{H}_{54} \mathrm{~N}_{2} \mathrm{O}_{3}$<smiles>CCCCCCCC(=O)NC(=CC(C)=CC=CC(C)=CC=CC=C(C)C=CC=C(C)C=CC1=C(C)CCCC1(C)C)C(=O)N(C)CCO</smiles>

M. Tomoaia-Cotisel, J. Zsako, E. Chifu, D.A. Ladenhead, Langmuir 1990, 6,191

44N $\beta$-apo-7'-benzoylamido-7'-carotenoic acid N,N-(bis(2-hydroxyethyl)-amide $\mathrm{C}_{43} \mathrm{H}_{56} \mathrm{~N}_{2} \mathrm{O}_{4}$<smiles>CCCCCC(=O)N(C)C(=CC(C)=CC=CC(C)=CC=CC=C(C)C=CC=C(C)C=CC1=C(C)CCCC1(C)C)C(=O)N(CCO)CCO</smiles>

M. Tomoaia-Cotisel, J. Zsako, E. Chifu, D.A. Ladenhead, Langmuir 1990, 6,191 
45N $\quad N$-octadecyl bixinamide<smiles>CCCCCCNC(=O)/C=C/C(C)=C/C=C/C(C)=C/C=C/C=C(C)/C=C/C=C(C)\C=C\C(=O)OC</smiles>

J.H. Fuhrhop, M. Krull, A. Schulz, D. Möbius, Langmuir 1990, 6,497. The authors list several related bixin amides.

G. Ferrari, V. Vecchietti, EP 030009, 1980 describe numerous bixin amides.

46N dibixine diphenylenediamid

$$
\mathrm{C}_{54} \mathrm{H}_{60} \mathrm{~N}_{2} \mathrm{O}_{6}
$$

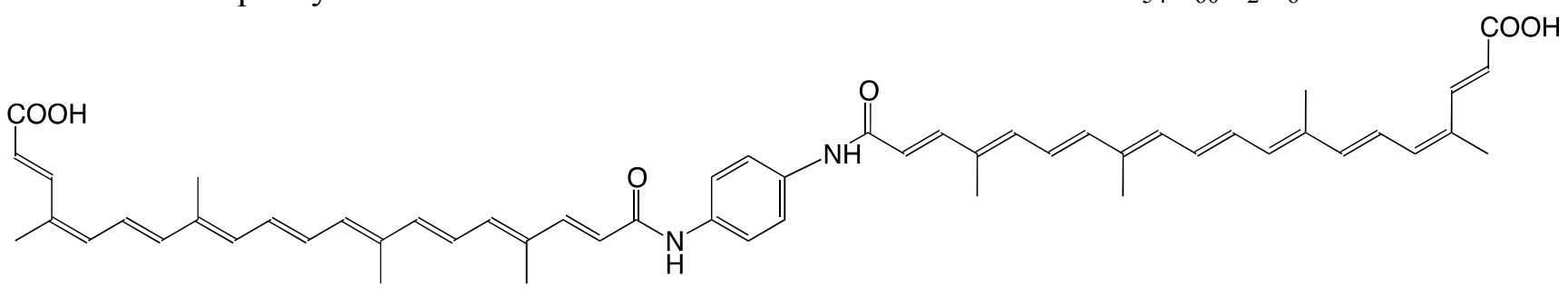

J.H. Fuhrhop, M. Krull, A. Schulz, D. Möbius, Langmuir 1990, 6, 497

$47 \mathrm{~N}$ imine of tris- $\left(8,8^{\prime}\right.$-diapo- $\psi, \psi$-carotene- $8.8^{\prime}$-diimine $)$

$\mathrm{C}_{72} \mathrm{H}_{96} \mathrm{~N}_{8}$

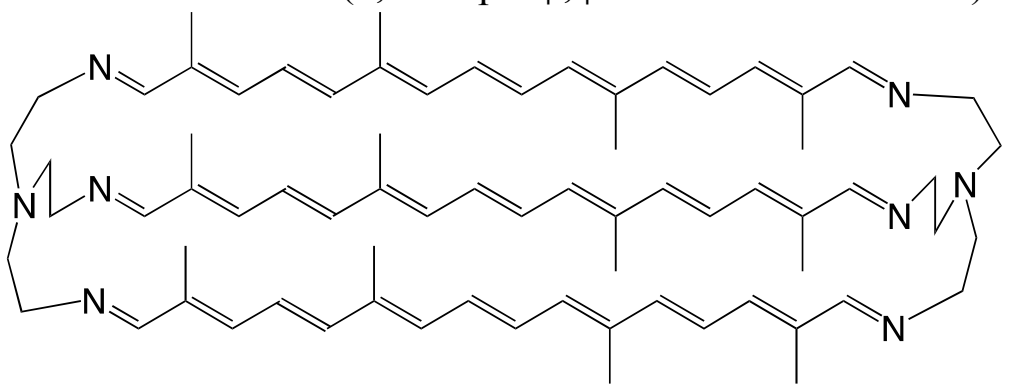

J.M. Lehn, J.P. Vigneron, I. Bkouhe-Waksman, J. Guilhem, C. Pascal, Helv. Chim. Acta 1992, 75, 1069 
$\phi$-carotenoids

48N 7'-aza-3'-methyl- $\beta$, $\phi$-carotene

$\mathrm{C}_{37} \mathrm{H}_{47} \mathrm{~N}$<smiles>CC1=C(/C=C/C(C)=C/C=C/C(C)=C/C=C/C=C(C)/C=C/C=C(C)/C=N/c2ccc(C)cc2)C(C)(C)CCC1</smiles>

H. Kamogawa, Polym. Lett. Ed. 1972, 10, 929

49N 7'-aza-3'-dimethylamino- $\beta, \phi$-carotene

$\mathrm{C}_{36} \mathrm{H}_{46} \mathrm{~N}_{2}$<smiles>C/C(C=Nc1ccc(N(C)C)cc1)=C\C=C\C(C)=C\C=C\C=C(C)\C=C\C=C(C)\C=C\C(C)=C1/C(C)CCCC1(C)C</smiles>

H. Ikeda, T. Sakai, Y. Kawabe, JP 2-2534, 1990

$50 \mathrm{~N} \quad 7$ '-aza- $\beta, \phi$-caroten-3'-amine

$\mathrm{C}_{36} \mathrm{H}_{46} \mathrm{~N}_{2}$<smiles>CC1=C(/C=C/C(C)=C/C=C/C(C)=C/C=C/C=C(C)/C=C/C=C(C)/C=N/c2ccc(N)cc2)C(C)(C)CCC1</smiles>

H. Ikeda, T. Sakai, Y. Kawabe, JP 2-2534, 1990

$51 \mathrm{~N} \quad 7$ '-aza-3'-methoxy- $\beta, \phi$-carotene

$\mathrm{C}_{37} \mathrm{H}_{47} \mathrm{NO}$<smiles>COc1ccc(/N=C/C(C)=C/C=C/C(C)=C/C=C/C=C(C)/C=C/C=C(C)/C=C/C2=C(C)CCCC2(C)C)cc1</smiles>

H. Ikeda, T. Sakai, Y. Kawabe, JP 2-2534, 1990 
$52 \mathrm{~N} \quad \beta, \phi$-carotene-3'-acetamide<smiles>CC(=O)Nc1ccc(/C=C/C(C)=C/C=C/C(C)=C/C=C/C=C(C)/C=C/C=C(C)/C=C/C2=C(C)CCCC2(C)C)cc1</smiles>

M.R. Wasielewski, P.A. Liddel, D. Barrett, T.A. Moore, D. Gust, Nature 1986, 322, 570

$53 \mathrm{~N} \quad \beta, \phi$-carotene-3'-porphyrinamide

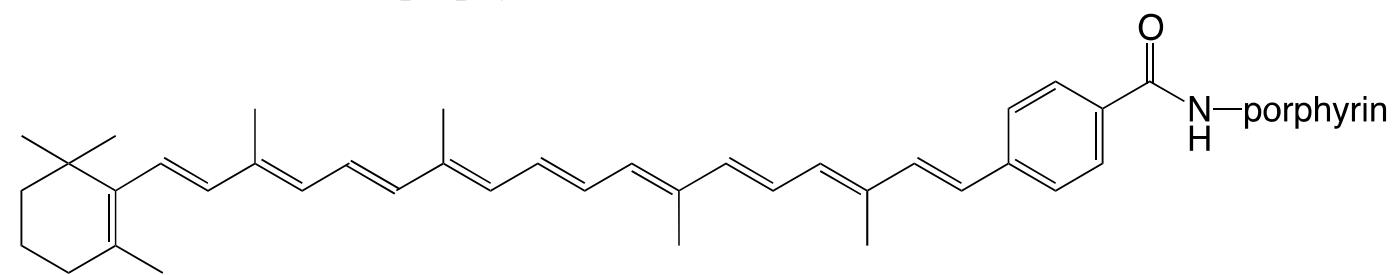

D. Gust, T.A. Moore, P.A. Liddell, G.A. Nemeth, L.R. Makings, A.L. Moore, D. Barrett, P.J. Pessiki, R.V. Bemasson, M. Rougiée, C. Chachaty, F.C. De Schryver, M. Van der Auweraer, A.R. Holzwarth, J. S. Connolly, J. Am. Chem. Soc. 1987, 109, 846

54N 3'-nitro- $\beta, \phi$-carotene<smiles>CC1=C(/C=C/C(C)=C/C=C/C(C)=C/C=C/C=C(C)/C=C/C=C(C)/C=C/c2ccc([N+](=O)[O-])cc2)C(C)(C)CCC1</smiles>

H. Ikeda, T. Sakai, Y. Kawabe, JP 2-2534, 1990

S. Gilmour, S.R. Marder, B.G. Tiemann, L.T. Cheng, J. Chem. Soc. Chem. Commun. 1993, 432 E.S. Hand, K.A. Belmore, L.D. Kispert, Helv. Chim. Acta 1993, 76, 1928

$55 \mathrm{~N} \quad 1$ ',3'-dinitro- $\beta, \phi$-carotene $\mathrm{C}_{37} \mathrm{H}_{44} \mathrm{~N}_{2} \mathrm{O}_{4}$<smiles>CC1=C(/C=C/C(C)=C/C=C/C(C)=C/C=C/C=C(C)/C=C/C=C(C)/C=C/c2ccc([N+](=O)[O-])cc2[N+](=O)[O-])C(C)(C)CCC1</smiles>

H. Ikeda, T. Sakai, Y. Kawabe, JP 2-2534, 1990 
$56 \mathrm{~N}$ 2-dimethylamino- $\phi, \phi$-carotene-3'-nitrile<smiles>CC(/C=C/C=C(C)/C=C/c1ccc(C#N)cc1)=C\C=C\C=C(C)\C=C\C=C(C)\C=C\c1ccc(N(C)C)cc1</smiles>

$\mathrm{C}_{37} \mathrm{H}_{40} \mathrm{~N}_{2}$

A. Slama-Schwok, M. Blanchard-Desce, J.M Lehn, J. Phys. Chem. 1990, 94, 3894

57N 3'-nitro- $\phi, \phi$-carotene-2-dimethylamine

$\mathrm{C}_{36} \mathrm{H}_{40} \mathrm{~N}_{2} \mathrm{O}_{2}$<smiles>CC(/C=C/C=C(C)/C=C/C(C)=C/C=C/c1ccc([N+](=O)[O-])cc1)=C\C=C\C(C)=C\C=C\c1cccc(N(C)C)c1</smiles>

A. Slama-Schwok, M. Blanchard-Desce, J.M Lehn, J. Phys. Chem. 1990, 94, 3894

58N 7'-cyano-3'-nitro- $\beta, \phi$-carotene

$\mathrm{C}_{38} \mathrm{H}_{44} \mathrm{~N}_{2} \mathrm{O}_{2}$<smiles>CC1=C(/C=C/C(C)=C/C=C/C(C)=C/C=C/C=C(C)/C=C/C=C(C)/C=C(\C#N)c2ccc([N+](=O)[O-])cc2)C(C)(C)CCC1</smiles>

H. Ikeda, T. Sakai, Y. Kawabe, JP 2-2534, 1990

59N 7'-cyano-7'-benzoxo- $\beta$-carotene

$\mathrm{C}_{39} \mathrm{H}_{45} \mathrm{NO}$<smiles>CC1=C(/C=C/C(C)=C/C=C/C(C)=C/C=C/C=C(C)/C=C/C=C(C)/C=C(\C#N)C(=O)c2ccccc2)C(C)(C)CCC1</smiles>

H. Ikeda, T. Sakai, Y. Kawabe, JP 2-2534, 1990

$60 \mathrm{~N} \quad 3$-dimethylamino- $\beta, \phi$-carotene

$\mathrm{C}_{39} \mathrm{H}_{51} \mathrm{~N}$<smiles>CC1=C(/C=C/C(C)=C/C=C/C(C)=C/C=C/C=C(C)/C=C/C=C(C)/C=C/c2ccc(N(C)C)cc2)C(C)(C)CCC1</smiles>

H. Ikeda, T. Sakai, Y. Kawabe, JP 2-2534, 1990 
$61 \mathrm{~N} \quad 3$-dioctylamino- $\beta, \phi$-carotene

$\mathrm{C}_{53} \mathrm{H}_{79} \mathrm{~N}$

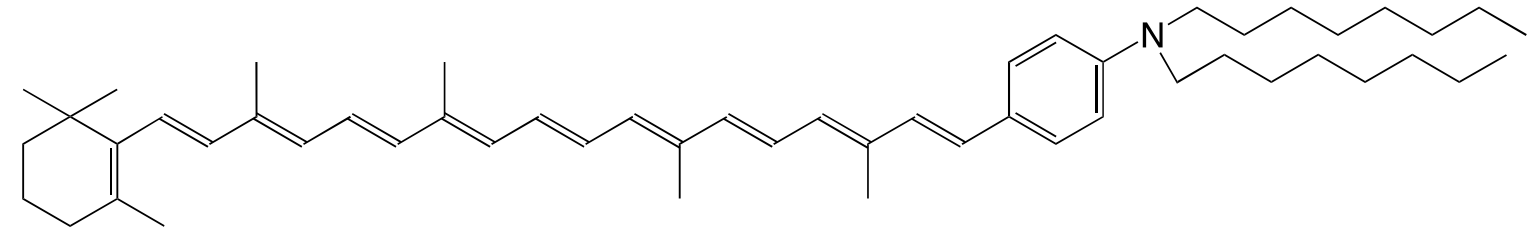

T. Wagner, S. Roth, Synth. Metals 1993, 54, 307

$62 \mathrm{~N} \quad 2$ ',3'-dicyano- $\beta, \phi$-carotene<smiles>CC1=C(/C=C/C(C)=C/C=C/C(C)=C/C=C/C=C(C)/C=C/C=C(C)/C=C/c2ccc(C#N)c(C#N)c2)C(C)(C)CCC1</smiles>

H. Ikeda, T. Sakai, Y. Kawabe, JP 2-2534, 1990

\subsection{Chalcogen-Carotenoids}

\subsubsection{Sulfur}

1S echinenone thione

$\mathrm{C}_{40} \mathrm{H}_{54} \mathrm{~S}$<smiles>CC1=C(/C=C/C(C)=C/C=C/C(C)=C/C=C/C=C(C)/C=C/C=C(C)/C=C/C2=C(C)C(=S)CCC2(C)C)C(C)(C)CCC1</smiles>

H.R. Sliwka, S. Liaaen-Jensen, Acta Chem. Scand. 1994, 48, 679

2S canthaxanthin thione

$\mathrm{C}_{40} \mathrm{H}_{52} \mathrm{OS}$

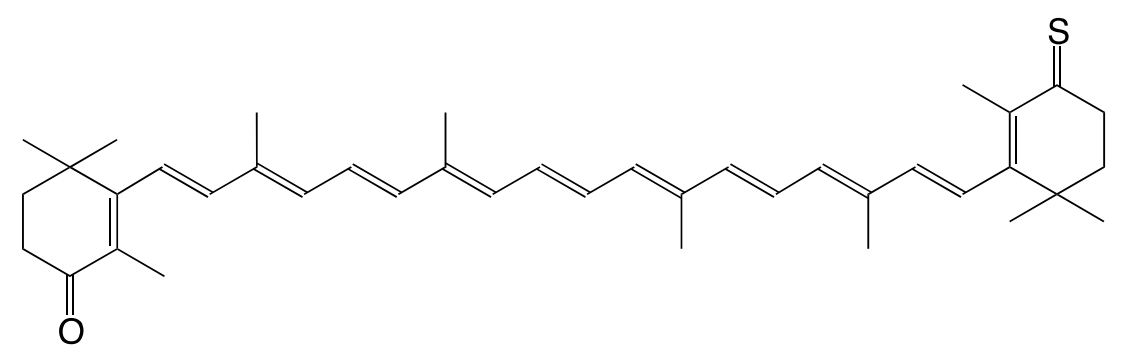

H.R. Sliwka, S. Liaaen-Jensen, Acta Chem. Scand. 1994, 48, 679 
3S rhodoxanthin thione

$\mathrm{C}_{40} \mathrm{H}_{50} \mathrm{OS}$<smiles>CC1=CC(=O)CC(C)(C)/C1=C\C=C(C)\C=C\C=C(C)\C=C\C=C(C)\C=C\C=C\C(C)=C\C=C1\C(C)=CC(=S)CC1(C)C</smiles>

H.R. Sliwka, S. Liaaen-Jensen, Acta Chem. Scand. 1994, 48, 679

4S 3'-thiolutein

$\mathrm{C}_{40} \mathrm{H}_{56} \mathrm{OS}$<smiles>CC1=CC(S)CC(C)(C)[C@H]1/C=C/C(C)=C/C=C/C(C)=C/C=C/C=C(C)/C=C/C=C(C)/C=C/C1=C(C)CCCC1(C)C</smiles>

H.R. Sliwka, S. Liaaen-Jensen, Acta Chem. Scand. 1990, 44, 61

$5 S \quad(3 S)-2$ ', 3'-didehydro- $\beta, \beta$-carotene-3-thiol

$$
\mathrm{C}_{40} \mathrm{H}_{54} \mathrm{~S}
$$<smiles>CC1=C(/C=C/C(C)=C/C=C/C(C)=C/C=C/C=C(C)/C=C/C=C(C)/C=C/C2=C(C)C[C@H](S)CC2(C)C)C(C)(C)C=CC1</smiles>

H.R. Sliwka, S. Liaaen-Jensen, Tetrahdron Asym. 1993, 4, 361

H.R. Sliwka, Helv. Chim. Acta 1999, 82, 161

$6 \mathrm{~S} \quad\left(3 R, 3^{\prime} S\right)-3$ '-sulfanyl- $\beta, \beta$-caroten-3-ol

$\mathrm{C}_{40} \mathrm{H}_{56} \mathrm{OS}$<smiles>CC1=C(/C=C/C(C)=C/C=C/C(C)=C/C=C/C=C(C)/C=C/C=C(C)/C=C/C2=C(C)C[C@H](S)CC2(C)C)C(C)(C)C[C@@H](O)C1</smiles>

H.R. Sliwka, S. Liaaen-Jensen, Tetrahdron Asym. 1993, 4, 361

H.R. Sliwka, Helv. Chim. Acta 1999, 82, 161 
7S 3'-thioacetyl lutein

$\mathrm{C}_{42} \mathrm{H}_{58} \mathrm{O}_{2} \mathrm{~S}$<smiles>CC1=C(/C=C/C(C)=C/C=C/C=C(C)/C=C/C=C(C)/C=C/C=C(C)/C=C/C2=C(C)C[C@@H](C(C)(C)C)CC2(C)C)C(C)(C)C[C@H](O)C1</smiles>

H.R. Sliwka, S. Liaaen-Jensen, Acta Chem. Scand. 1990, 44, 61

8S 4,4'-dithioacetyl- $\beta, \beta$-carotene

$\mathrm{C}_{44} \mathrm{H}_{60} \mathrm{O}_{2} \mathrm{~S}_{2}$<smiles>CC(C=CC=C(C)C=CC=C(C)C=CC1=C(C)C(C(C)(C)C)CCC1(C)C)=CC=CC=C(C)C=CC1=C(C)C(C(C)(C)C)CCC1(C)C</smiles>

H.R. Sliwka, Helv. Chim. Acta 1999, 82, 161

9S 4'-thioacethyl- $\beta, \beta$-caroten-4-one

$\mathrm{C}_{42} \mathrm{H}_{56} \mathrm{O}_{2} \mathrm{~S}$<smiles>CC1=C(/C=C/C(C)=C/C=C/C(C)=C/C=C/C=C(C)/C=C/C=C/C2=C(C)C([As](C)(=O)=O)CCC2(C)C)C(C)(C)CCC1=O</smiles>

H.R. Sliwka, S. Liaaen-Jensen, Acta Chem. Scand. 1990, 44, 61

10S (3R)-3',4'-didehydro-3-phenylsulfanyl- $\beta, \beta$-carotene

$$
\mathrm{C}_{46} \mathrm{H}_{58} \mathrm{~S}
$$<smiles>CCCCCCCCC1=C(/C=C/C(C)=C/C=C/C(C)=C/C=C/C=C(C)/C=C/C=C(C)/C=C/C2C(C)C=CCC2(C)C)C(C)(C)C[C@@H]([SH](C)CCCCC)C1</smiles>

J. Inananga, M. Yamaguchi, Mem. Fac. Sci. Kyushi Univ. Ser. C, 1989, 17, 109 
$11 \mathrm{~S} \quad 3,3$ '-diphenylsulfanyl- $\beta, \beta$-carotene

$\mathrm{C}_{52} \mathrm{H}_{64} \mathrm{~S}_{2}$

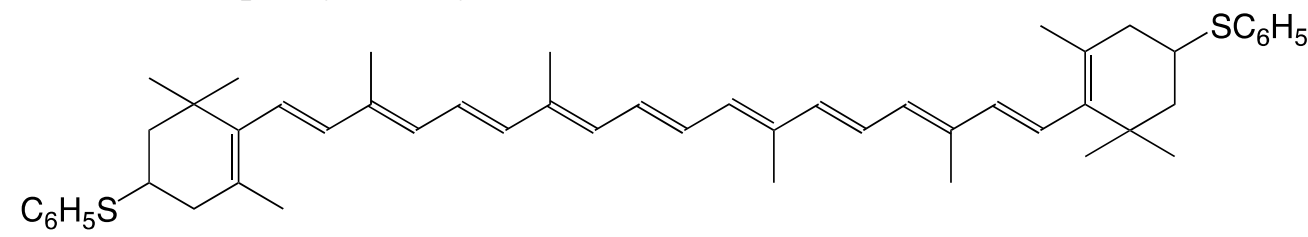

J. Inananga, M. Yamaguchi, Mem. Fac. Sci. Kyushi Univ. Ser. C, 1989, 17, 109

12S 4,4'-diphenylsulfanyl- $\beta, \beta$-carotene

$\mathrm{C}_{52} \mathrm{H}_{64} \mathrm{~S}_{2}$

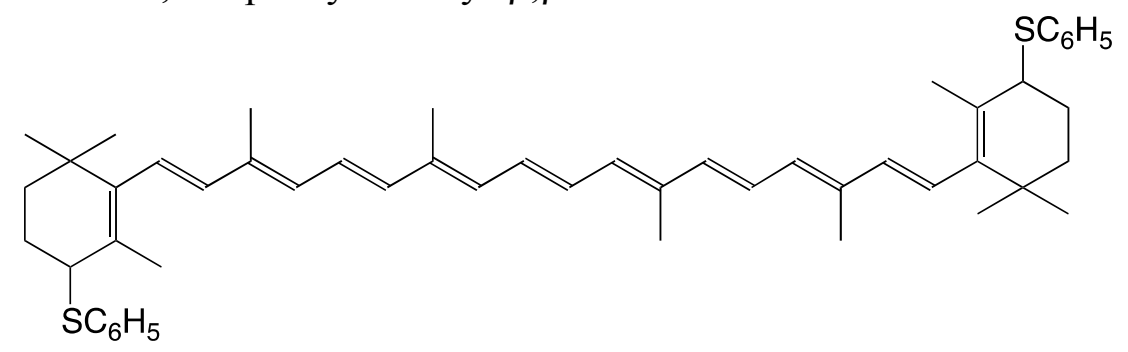

C. Martin, P. Karrer, Helv. Chim. Acta 1959, 42, 464

$13 \mathrm{~S} \quad 3,4$ '-dehydro- $\beta, \beta$-carotene-4-thioglucopyranoside

$\mathrm{C}_{74} \mathrm{H}_{80} \mathrm{O}_{9} \mathrm{~S}$

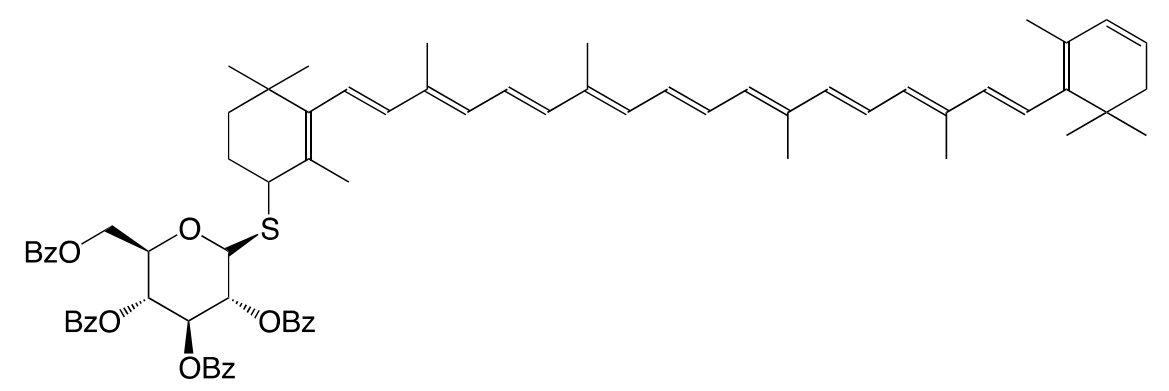

V. Nagy, A. Agócs, E. Turcsi, J. Deli, Tetrahedron Lett. 2010, 52, 1020

14S $\beta, \beta$-carotene-4,4'-bisthioglucopyranoside

$\mathrm{C}_{107} \mathrm{H}_{106} \mathrm{O}_{18} \mathrm{~S}_{2}$

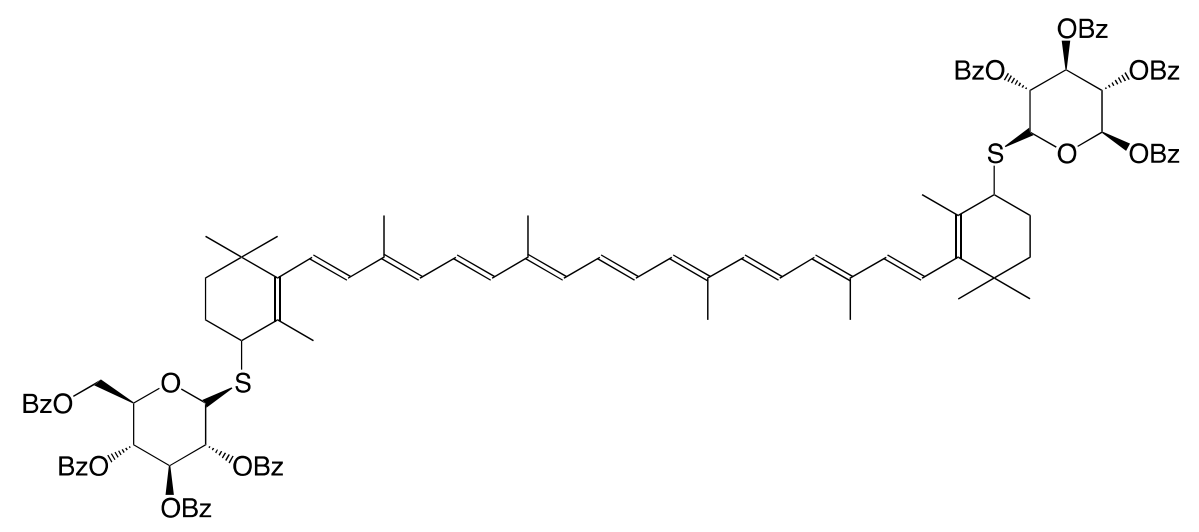

V. Nagy, A. Agócs, E. Turcsi, J. Deli, Tetrahedron Lett. 2010, 52, 1020 
15S $\beta, \beta$-carotene-15-yl-phenylsulfone

$\mathrm{C}_{46} \mathrm{H}_{60} \mathrm{O}_{2} \mathrm{~S}$<smiles>CC(=CC=C/C(C)=C/C(=C\C=C(C)\C=C\C=C(C)\C=C\C1=C(C)CCCC1(C)C)S(C)(=O)=O)/C=C/C1=C(C)CCCC1(C)C</smiles>

K. Berhard, S. Jäggli, P. Kreienbühl, U. Schwieter, EU298404, 1989. The patent lists numerous other phenylsulfones and $p$-chlorophenylsulfones as synthetic intermediates.

$\phi$-carotenoids

16S 3-methylsulfanyl-8'-apo- $\phi$-carotenal

$\mathrm{C}_{28} \mathrm{H}_{32} \mathrm{OS}$<smiles>CC(C=O)=CC=CC(C)=CC=CC=C(C)C=CC=C(C)C=Cc1ccc(S(C)(=O)=O)cc1</smiles>

Y.Q. Shen, W. Göhring, S. Hagen, S. Roth, J. Mol. Electron. 1990, 6, 31

17S $\beta, \phi$-carotene-3'-methanethiol

$\mathrm{C}_{38} \mathrm{H}_{48} \mathrm{~S}$<smiles>CC1=C(/C=C/C(C)=C/C=C/C(C)=C/C=C/C=C(C)/C=C/C=C/c2ccc(CS)cc2)C(C)(C)CCC1</smiles>

G. Leatherman, E.N. Duranti, D. Gust, T.A. Moore, A.L. Moore, S. Stone, Z. Zhou, P. Rez, Y.Z. Liu, S.M. Lindsay, J. Phys. Chem. B 1999, 103, 4006

$18 \mathrm{~S} \quad 10,10$ '-dimethyl-13-phenyl-9,9',13,13'-tetranor- $\phi, \phi$-carotene-3,3'-dimethanethiol $\mathrm{C}_{40} \mathrm{H}_{40} \mathrm{~S}_{2}$<smiles>CSc1ccc(/C=C/C=C(C)/C=C/C=C/C=C/C=C(/C=C/C(C)=C/C=C/c2ccc(SC)cc2)c2ccccc2)cc1</smiles>

J. Maeng, S.B. Kim, N.J. Lee, E. Choi, S.Y. Jung, I. Hong, S.H. Bae, J.T. Oh, B. Lim, J.W. Kim, C.J. Kang, S. Koo, Chem. Eur. J. 2010, 16, 7395. The authors list other similar compounds. 
1Se (3S)-2',3'-didehydro- $\beta, \beta$-carotene-3-phenylselenide

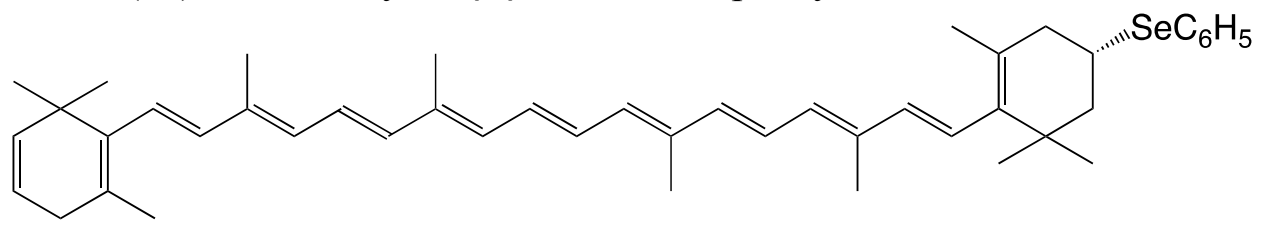

$\mathrm{C}_{46} \mathrm{H}_{58} \mathrm{Se}$

H.R. Sliwka, S. Liaaen-Jensen, Acta Chem. Scand. 1995, 49, 428

E. Oliveros, A.M. Braun, T. Aminian-Saghafi, H.R. Sliwka, New J. Chem. 1994, 18, 535

H.R. Sliwka, Helv. Chim. Acta 1999, 82, 161

2Se zeaxanthin phenylselenide, $(3 R, 3 ' S)$-3'-phenylseleno- $\beta, \beta$-caroten-3-ol

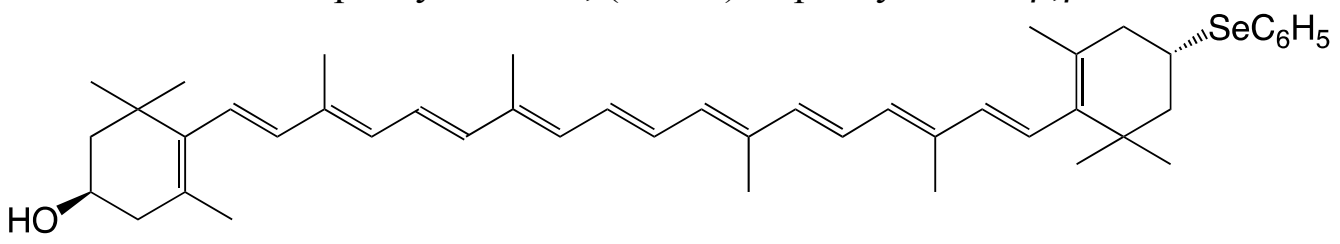

H.R. Sliwka, S. Liaaen-Jensen, Acta Chem. Scand. 1995, 49, 428

H.R. Sliwka, Helv. Chim. Acta 1999, 82, 161

3Se zeaxanthin diphenylselenide, $(3 S, 3$ 'S)- $\beta, \beta$-carotene-3,3'-diphenylselenide $\mathrm{C}_{52} \mathrm{H}_{64} \mathrm{Se}_{2}$

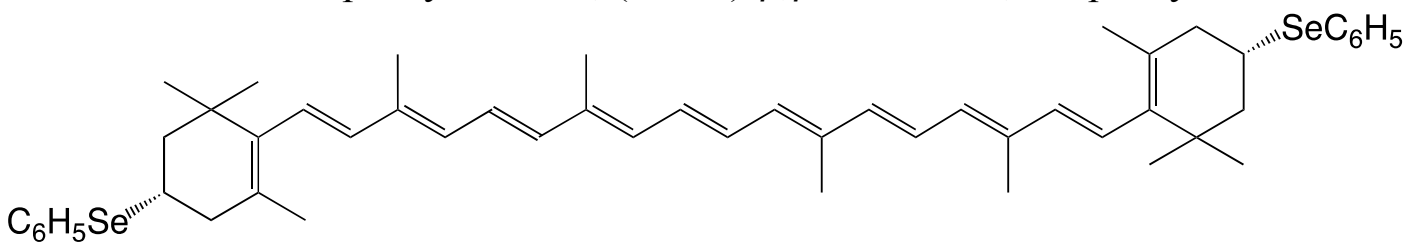

H.R. Sliwka, S. Liaaen-Jensen, Acta Chem. Scand. 1995, 49, 428

H.R. Sliwka, Helv. Chim. Acta 1999, 82, 161

4Se lutein phenylselenide, $\left(3 R, 3^{\prime} R, S, 6^{\prime} R\right)$-3'-phenyleleno- $\beta, \varepsilon$-caroten-3-ol

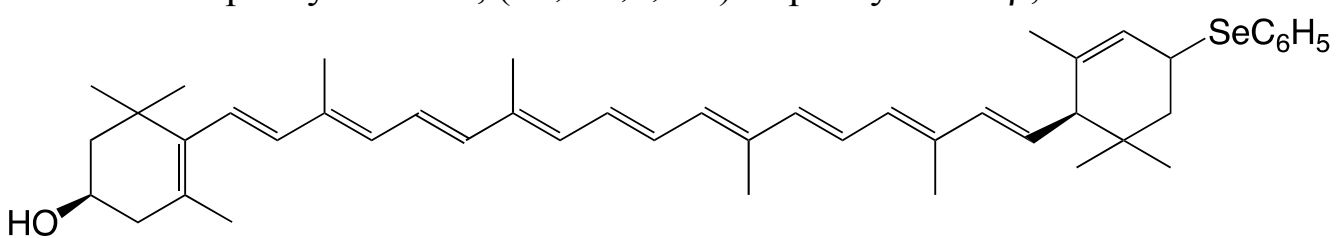

H.R. Sliwka, S. Liaaen-Jensen, Acta Chem. Scand. 1995, 49, 428 
5Se rhodoxanthin diphenylselenide, 7,7'-di(phenylseleno)-7,8,7',8'-dihydro-retro- $\varepsilon, \varepsilon$-carotene3,3'-dione $\mathrm{C}_{52} \mathrm{H}_{62} \mathrm{O}_{2} \mathrm{Se}_{2}$

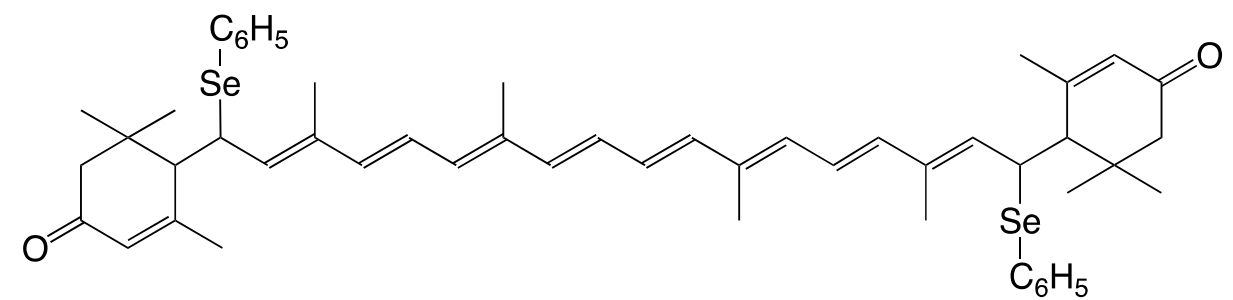

postulated unstable intermediate

H.R. Sliwka, S. Liaaen-Jensen, Acta Chem. Scand. 1995, 49, 856

6Se $\quad(3 S)-2$ ',3'-didehydro- $\beta, \beta$-caroten-3-yl-di-O,O-propylselenophosphate

$\mathrm{C}_{46} \mathrm{H}_{67} \mathrm{O}_{3} \mathrm{PSe}$<smiles>CCCOP(=O)(OCCC)O[C@H]1CC(C)=C(/C=C/C(C)=C/C=C/C(C)=C/C=C/C=C(C)/C=C/C=C(C)/C=C/C2=C(C)CC=CC2(C)C)C(C)(C)C1</smiles>

H.R. Sliwka, Acta Chem. Scand. 1997, 51, 345

H.R. Sliwka, Helv. Chim. Acta 1999, 82, 161

7Se $\quad(3 R, 3 ' S)$-3-hydroxy-3-yl-di-O,O-propylselenophosphate

$\mathrm{C}_{46} \mathrm{H}_{69} \mathrm{O}_{4} \mathrm{PSe}$<smiles>CCCOP(=O)(OCCC)O[C@H]1CC(C)=C(/C=C/C(C)=C/C=C/C(C)=C/C=C/C=C(C)/C=C/C=C(C)/C=C/C2=C(C)C[C@@H](O)CC2(C)C)C(C)(C)C1</smiles>

H.R. Sliwka, Acta Chem. Scand. 1997, 51, 345

H.R. Sliwka, Helv. Chim. Acta 1999, 82, 161 


\subsection{Combinations}

$1 \mathrm{NS}$ zeaxanthin thiocyanate, $(3 S)$-3'-thiocyano- $\beta, \beta$-caroten-3-ol

$\mathrm{C}_{41} \mathrm{H}_{55} \mathrm{NOS}$<smiles>CC1=C(/C=C/C(C)=C/C=C/C(C)=C/C=C/C=C(C)/C=C/C=C(C)/C=C/C2=C(C)C[C@@H](C#N)CC2(C)C)C(C)(C)C[C@H](O)C1</smiles>

H.R. Sliwka, S. Liaaen-Jensen, Tetrahedron Asym. 1993, 4, 2377

2NS zeaxanthin dithiocyanat, $(3 S, 3$ 'S)- $\beta, \beta$-carotene-3,3'-dithiocyanate

$\mathrm{C}_{42} \mathrm{H}_{54} \mathrm{~N}_{2} \mathrm{~S}_{2}$<smiles>CC1=C(/C=C/C(C)=C/C=C/C(C)=C/C=C/C=C(C)/C=C/C=C(C)/C=C/C2=C(C)C[C@@H](S(N)(=O)=O)CC2(C)C)C(C)(C)C[C@H](S)C1</smiles>

H.R. Sliwka, S. Liaaen-Jensen, Tetrahedron Asym. 1993, 4, 2377

6.5. Iron

$\mathrm{Fe}$

$1 \mathrm{Fe}$ ferrocenyl-C21 aldehyde, 7-ferrocenyl-7,8'-diapocaroten-8'-al

$\mathrm{C}_{31} \mathrm{H}_{35} \mathrm{FeO}$

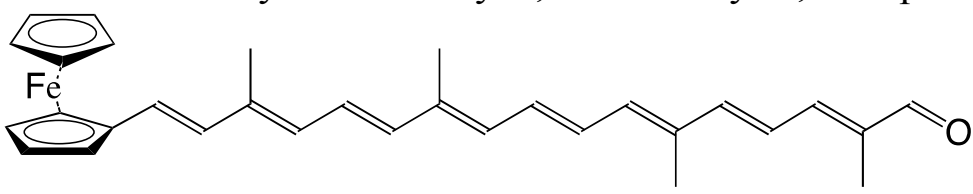

F. Effenberger, H. Schlosser, Synthesis 1990, 1085

2Fe ferrocenyl-C31 aldehyde

$\mathrm{C}_{41} \mathrm{H}_{47} \mathrm{FeO}$

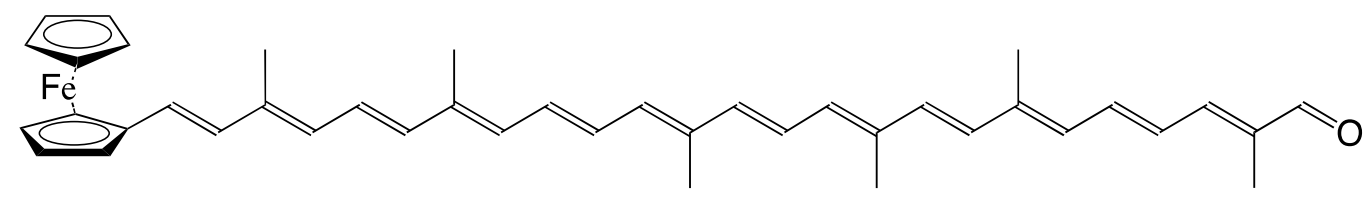

F. Effenberger, H. Schlosser, Synthesis 1990, 1085 
3Fe C22 bis(ferrocenyl), 7,7'-bis(ferrocenyl)-7,7'-diapocarotene

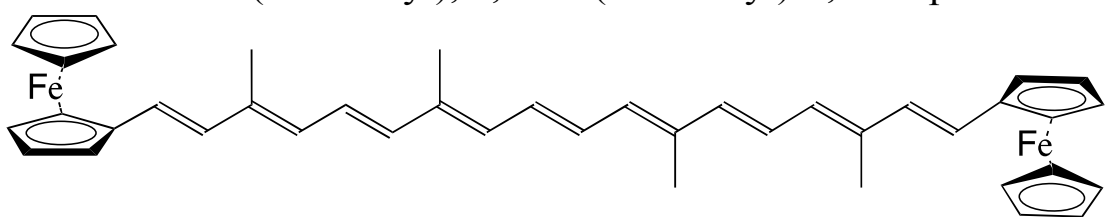

F. Effenberger, H. Schlosser, Synthesis 1990, 1085

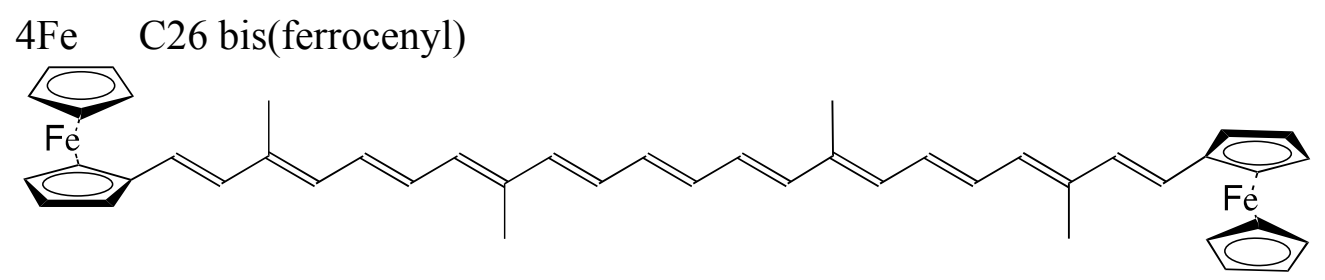

$\mathrm{C}_{46} \mathrm{H}_{52} \mathrm{Fe}_{2}$

J.M. Lehn, Angew. Chem. Int. Ed. 1990, 29, 1304

$5 \mathrm{Fe} \quad$ tetrakis(iron tricarbonyl)- $\beta, \beta$-carotene

$\mathrm{C}_{52} \mathrm{H}_{56} \mathrm{Fe}_{4} \mathrm{O}_{12}$

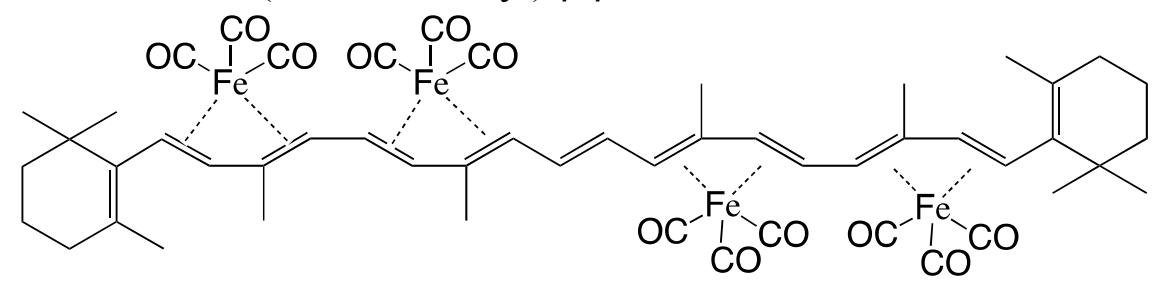

M. Ichikawa, M. Tsutsui, F. Vohwinkel, Z. Naturforschg. 1967, 22b, 376

$6 \mathrm{Fe}$ tetrakis(iron tricarbonyl)- $\beta, \beta$-carotene

$\mathrm{C}_{52} \mathrm{H}_{56} \mathrm{Fe}_{4} \mathrm{O}_{12}$

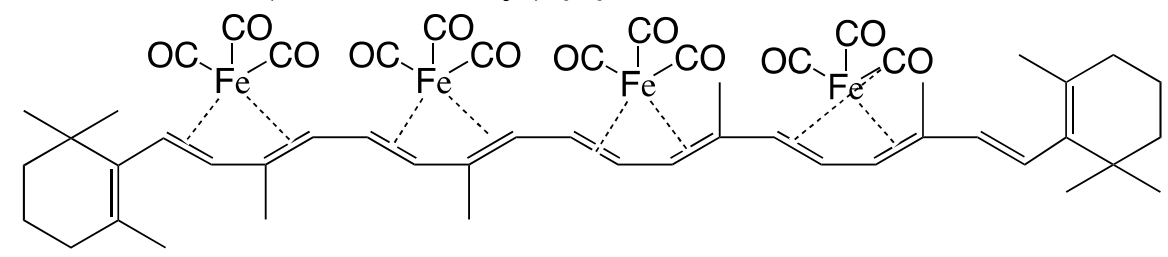

M. Ichikawa, M. Tsutsui, F. Vohwinkel, Z. Naturforschg. 1967, 22b, 376 
$7 \mathrm{Fe} \quad$ tetrakis(iron tricarbonyl)- $\beta, \beta$-carotene

$\mathrm{C}_{52} \mathrm{H}_{56} \mathrm{Fe}_{4} \mathrm{O}_{12}$

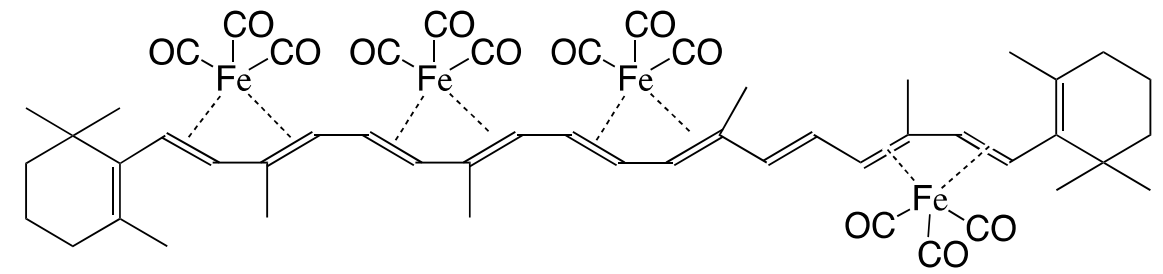

M. Ichikawa, M. Tsutsui, F. Vohwinkel, Z. Naturforschg. 1967, 22b, 376

\subsection{Heterocycle Carotenoids}

6.6.1. N-heterocycle

○N

$1 \odot \mathrm{N}$ crocetin-di-imidazolide

$\mathrm{C}_{26} \mathrm{H}_{30} \mathrm{~N}_{4} \mathrm{O}_{2}$<smiles>CC(=C/C=C/C(C)=C/C=C/C=C(C)/C=C/C=C(\C)C(=O)n1ccnc1)C(=O)n1ccnc1</smiles>

H. Pfander, F. Wittwer, Helv. Chim. Acta 1979, 62, 1944

$2 \odot \mathrm{N}$ crocetin-bis(1,2,4-triazolide)

$\mathrm{C}_{26} \mathrm{H}_{30} \mathrm{~N}_{4} \mathrm{O}_{2}$<smiles>CC(=C/C=C/C(C)=C/C=C/C=C(C)/C=C/C=C(\C)C(=O)n1ccnn1)C(=O)n1cncn1</smiles>

H. Pfander, F. Wittwer, Helv. Chim. Acta 1979, 62, 1944

$3 \odot \mathrm{N}$ octadexylbixin imidazolide

$\mathrm{C}_{45} \mathrm{H}_{67} \mathrm{~N}_{3} \mathrm{O}_{2}$

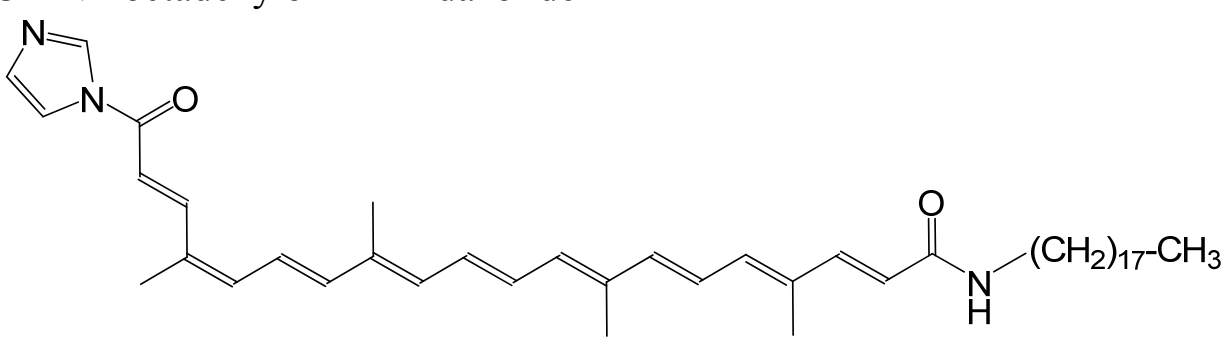

J.H. Fuhrhop, M. Krull, A. Schulz, D. Möbius, Langmuir 1990, 6,497 
$4 \odot \mathrm{N} \quad N$-(8'-apo- $\beta$-carotene-8-ylidene)-pyrrolidium perchlorate

$\mathrm{C}_{34} \mathrm{H}_{48} \mathrm{ClNO}_{4}$<smiles>CC1=C(/C=C/C(C)=C/C=C/C(C)=C/C=C/C=C(C)/C=C/C=C(\C)C=[N+]2CCCC2)C(C)(C)CCC1</smiles>

D.L. Coffen, E. Ho, C. Nocka, G. Sasso, V. Toome, T.R. Wagler, T.H. Williams, J. Prakt. Chem. 1993, 335, 135

$5 \odot \mathrm{N} \quad$ 7'-apo-7'-( $N$-methyl-4-pyridinium)- $\beta$-carotene iodide

$\mathrm{C}_{37} \mathrm{H}_{48} \mathrm{IN}$<smiles>CC1=C(/C=C/C(C)=C/C=C/C(C)=C/C=C/C=C(C)/C=C/C=C(C)/C=C/C2=CC=[N+](C)[I-]=C2)C(C)(C)CCC1</smiles>

H. Ikeda, T. Sakai, Y. Kawabe, JP 2-2534, 1990

$6 \odot \mathrm{N} \quad$ 7'-apo-7'-( $N$-methyl-2-pyridinium) $-\beta$-carotene iodide

$\mathrm{C}_{37} \mathrm{H}_{48} \mathrm{IN}$<smiles>CC1=C(/C=C/C(C)=C/C=C/C(C)=C/C=C/C=C(C)/C=C/C=C(C)/C=C/c2cccc[n+]2C)C(C)(C)CCC1</smiles>

H. Ikeda, T. Sakai, Y. Kawabe, JP 2-2534, 1990

7๑N 7,7'-diapo-7,7'-bis(2-pyridyl)-carotene

$\mathrm{C}_{32} \mathrm{H}_{34} \mathrm{~N}_{2}$<smiles>CC(/C=C/C=C(C)/C=C/c1ccccn1)=C\C=C\C=C(C)\C=C\C=C(C)\C=C\c1ccccn1</smiles>

H.R. Brahmana, K. Katsuyama, J. Inaga, T. Katsuki, M. Yamaguchi, Tetrahedron Lett. 1981, 22, 1695

$8 \odot \mathrm{N} \quad$ 7,7'-diapo-7,7'-bis(3-pyridyl)-carotene

$\mathrm{C}_{32} \mathrm{H}_{34} \mathrm{~N}_{2}$<smiles>CC(/C=C/C=C/c1cccnc1)=C\C=C\C=C(C)\C=C\C=C(C)\C=C\c1cccnc1</smiles>

H.R. Brahmana, K. Katsuyama, J. Inaga, T. Katsuki, M. Yamaguchi, Tetrahedron Lett. 1981, 22, 1695 T.S. Arrhenius, M. Blanchard-Desce, M. Dvolaitzky, J.M. Lehn, J. Malthête, Proc. Natl. Acad. Sci. USA 1986, 83, 5355 
$9 \odot \mathrm{N} \quad$ 7,7'-diapo-7,7'-bis(4-pyridyl)-carotene<smiles>CC(/C=C/C=C(C)/C=C/C=C(C)/C=C/c1ccncc1)=C\C=C\C(C)=C\C=C\c1ccncc1</smiles>

$\mathrm{C}_{32} \mathrm{H}_{34} \mathrm{~N}_{2}$

I. Visoly-Fisher, K. Daie, Y. Terazono, C. Herrero, F. Fungo, L. Otero, E. Durantini, J.J. Silber, L. Sereno, D. Gust, T.A. Moore, A.L. Morre, S.M. Lindsay, PNAS 2006, 103, 8686

$10 \odot \mathrm{N} \quad$ bis(4-pyridyl)-C26:11-carotene $\mathrm{C}_{36} \mathrm{H}_{38} \mathrm{~N}_{2}$<smiles>CC(/C=C/C=C/C=C/c1ccncc1)=C\C=C\C=C(C)\C=C\C=C(C)\C=C\C=C\c1ccncc1</smiles>

I. Visoly-Fisher, K. Daie, Y. Terazono, C. Herrero, F. Fungo, L. Otero, E. Durantini, J.J. Silber, L. Sereno, D. Gust, T.A. Moore, A.L. Morre, S.M. Lindsay, PNAS 2006, 103, 8686

$11 \odot \mathrm{N} \quad$ bis(4-pyridyl)-C26:11-carotene $\mathrm{C}_{36} \mathrm{H}_{38} \mathrm{~N}_{2}$<smiles>CC(/C=C/C=C/C=C/C(C)=C/C=C/C=C(C)/C=C/c1ccncc1)=C\C=C\C=C(C)\C=C\c1ccncc1</smiles>

T.S. Arrhenius, M. Blanchard-Desce, M. Dvolaitzky, J.M. Lehn, J. Malthête, Proc. Natl. Acad. Sci. USA 1986, 83, 5355

$12 \odot \mathrm{N} \quad$ bis(4-pyridyl)-C34:15-carotene<smiles>CC(/C=C/C=C/C=C/C(C)=C/C=C/C=C(C)/C=C/c1ccncc1)=C\C=C\C=C(C)\C=C\c1ccncc1</smiles>

$\mathrm{C}_{36} \mathrm{H}_{38} \mathrm{~N}_{2}$

T.S. Arrhenius, M. Blanchard-Desce, M. Dvolaitzky, J.M. Lehn, J. Malthête, Proc. Natl. Acad. Sci. USA 1986, 83, 5355 
$13 \odot \mathrm{N} \quad$ 1,4-bis(4-pyridyl-C12:5)-benzene

$\mathrm{C}_{40} \mathrm{H}_{40} \mathrm{~N}_{2}$<smiles>CC(/C=C/C=C/C=C(C)/C=C/c1ccncc1)=C\C=C\C=C(C)\C=C\c1ccc(/C=C/c2ccncc2)cc1</smiles>

T.S. Arrhenius, M. Blanchard-Desce, M. Dvolaitzky, J.M. Lehn, J. Malthête, Proc. Natl. Acad. Sci. USA 1986, 83, 5355

$14 \odot \mathrm{N} \quad$ 1,4-bis(4-pyridinium-C12:5)-benzene diiodide

$\mathrm{C}_{42} \mathrm{H}_{46} \mathrm{I}_{2} \mathrm{~N}_{2}$<smiles>CC(/C=C/C=C(C)/C=C/c1cc[n+](C)cc1)=C\C=C\C=C(C)\C=C\c1ccc(/C=C/C(C)=C/C=C/c2cc[n+](C)cc2)cc1</smiles>

T.S. Arrhenius, M. Blanchard-Desce, M. Dvolaitzky, J.M. Lehn, J. Malthête, Proc. Natl. Acad. Sci. USA 1986, 83, 5355

$15 \odot \mathrm{N} \quad$ 7,7'-diapo-7,7'-bis(4-methylpyridinium)-carotene diiodide $\mathrm{C}_{34} \mathrm{H}_{40} \mathrm{I}_{2} \mathrm{~N}_{2}$<smiles>CC(/C=C/C=C/c1cc[n+](C)cc1)=C\C=C\C(C)=C\C=C\C(C)=C\C=C\C(C)=C\c1cc[n+](C)cc1</smiles>

T.S. Arrhenius, M. Blanchard-Desce, M. Dvolaitzky, J.M. Lehn, J. Malthête, Proc. Natl. Acad. Sci. USA 1986, 83, 5355

$16 \odot \mathrm{N} \quad 7,7^{\prime}$-diapo-7,7'-bis(4-ethylpyridinium)-carotene dibromide $\mathrm{C}_{36} \mathrm{H}_{44} \mathrm{Br}_{2} \mathrm{~N}_{2}$<smiles>CC[n+]1ccc(/C=C/C(C)=C/C=C/C(C)=C/C=C/C=C(C)/C=C/C=C(C)/C=C/C23C=C[N+](CC)(CC2)CC3)cc1</smiles>

T. Okumoto, N. Morita, I. Nakamura, M. Konishi, M. Yamaguchi, J. Cancer Res.. Clin. Oncol. 1985, 109,257 
$17 \odot \mathrm{N} \quad$ bis(4-pyridinium)-C34:15-carotene diiodide<smiles>CC(/C=C/C=C/C=C(C)/C=C/c1c[c-][n+](C)cc1)=C\C=C\C=C\C(C)=C\C=C\C=C(C)\C=C\c1cc[n+](C)cc1</smiles>

$\mathrm{C}_{38} \mathrm{H}_{44} \mathrm{I}_{2} \mathrm{~N}_{2}$

T.S. Arrhenius, M. Blanchard-Desce, M. Dvolaitzky, J.M. Lehn, J. Malthête, Proc. Natl. Acad. Sci. USA 1986, 83, 5355

$18 \odot \mathrm{N} \quad 7,7^{\prime}$-diapo-7,7'-bis(1,3,3 trimethylindolenium)-carotene dichlorate

$\mathrm{C}_{42} \mathrm{H}_{48} \mathrm{Cl}_{2} \mathrm{~N}_{2} \mathrm{O}_{8}$<smiles>CC(/C=C/C=C(C)/C=C/C=C(C)/C=C/C1=[N+](C)c2ccccc2C1(C)C)=C\C=C\C=C(C)\C=C\C=C(/C)C1=[N+](C)c2ccccc2C1</smiles>

S. Hünig, F. Linhart, D. Scheutzow, Liebigs Ann. 1975, 2089

$19 \odot \mathrm{N} \quad$ 7,7'-diapo-7'-(4-pyridyl)- $\phi$-carotene-2-dimethylamine $\mathrm{C}_{35} \mathrm{H}_{40} \mathrm{~N}_{2}$<smiles>CC(/C=C/C=C(C)/C=C/c1ccncc1)=C\C=C\C=C(C)\C=C\C=C(C)\C=C\c1cccc(N(C)C)c1</smiles>

M. Blanchard-Desce, J.M. Lehn, I. Ledoux, J. Zyss, Special Publication - Royal Society of Chemistry (Org. Mater. Non-linear Opt.) 1989, 69, 170

$20 \odot \mathrm{N} \quad$ 7'-cyano-7'-(4-pyridyl)- $\beta, \phi$-carotene

$$
\mathrm{C}_{37} \mathrm{H}_{44} \mathrm{~N}_{2}
$$<smiles>CC1=C(/C=C/C(C)=C/C=C/C(C)=C/C=C/C=C(C)/C=C/C=C(C)/C=C(\C#N)c2ccncc2)C(C)(C)CCC1</smiles>

A.J. Cruz, K. Siam, D.P. Rillema, J. Phys. Chem. 2011, 115, 1108 
$21 \odot \mathrm{N}$ lutein- $6 H-1,2$-oxazine

$\mathrm{C}_{39} \mathrm{H}_{53} \mathrm{NO}_{3}$<smiles>CC1=C[C@H](O)CC(C)(C)[C@H]1/C=C/C(C)=C/C=C/C(C)=C/C=C/C=C(C)/C=C/C=C(\C)C1=NOC2C[C@H](O)CC(C)(C)C2=C1</smiles>

M. Tsuboi, H. Etoh, Y. Yomoda, K. Kato, H. Kato, A. Kulkarni, Y. terada, T. Maoka, H. Mori, T. Inakuma, Tetrahedron Lett. 2010, 521, 676

$22 \odot \mathrm{N} \quad 6,6$ '-diapocarotenal-6'-(2-phenyl-azlactone)

$\mathrm{C}_{33} \mathrm{H}_{33} \mathrm{NO}_{3}$<smiles>c1ccccc1</smiles><smiles>C/N=C(C)\C=C\C=C(C)\C=C\C=C(C)\C=C\C=C\C=C(C)\C=C\C=C(C)\C=C\C=O</smiles>

J. Zsako, M. Tomoaia-Cotisel, V. Tamas, C. Coman, E. Chifu, Rev. Roum. Chim. 1987, 32, 1193. The authors describe several other azlactones.

$23 \odot \mathrm{N} \quad \beta$-apo-6'-carotenal-2-phenyl-azlactone $\mathrm{C}_{39} \mathrm{H}_{45} \mathrm{NO}_{2}$<smiles>CC1=C(/C=C/C(C)=C/C=C/C(C)=C/C=C/C=C(C)/C=C/C=C(C)/C=C2\N=C(c3ccccc3)OC2=O)C(C)(C)CCC1</smiles>

V. Tamas, V. Ciurdaru, C. Bodea, Rev. Roum. Chim. 1973, 18, 1409

$24 \odot \mathrm{N} \quad \beta$-apo-6'-carotendial bis-2-phenyl diazlactone, C24:11 diazlactone $\mathrm{C}_{42} \mathrm{H}_{38} \mathrm{~N}_{2} \mathrm{O}_{4}$ 1<smiles>CC1=N/C(=C\C=C\C(C)=C\C=C\C=C(C)\C=C\C=C(C)\C=C\C=C\C=C\C=C2\N=C(C)OC2=O)C(=O)O1</smiles>

V. Tamas, V. Ciurdaru, C. Bodea, Rev. Roum. Chim. 1973, 18, 1409 
$25 \odot \mathrm{N} \quad \mathrm{C} 30: 13$-diazlactone

$\mathrm{C}_{48} \mathrm{H}_{48} \mathrm{~N}_{2} \mathrm{O}_{4}$<smiles>c1ccccc1</smiles>

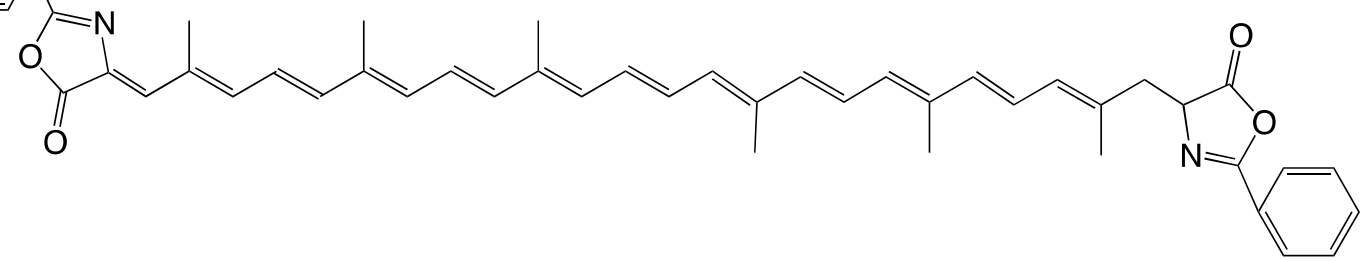

V. Tamas, V. Ciurdaru, C. Bodea, Rev. Roum. Chim. 1973, 18, 1409. The authors describe several other azlactones.

$26 \odot \mathrm{N} \quad$ bis(2,2'-bipyridine-4-yl)-carotene

$\mathrm{C}_{42} \mathrm{H}_{40} \mathrm{~N}_{4}$

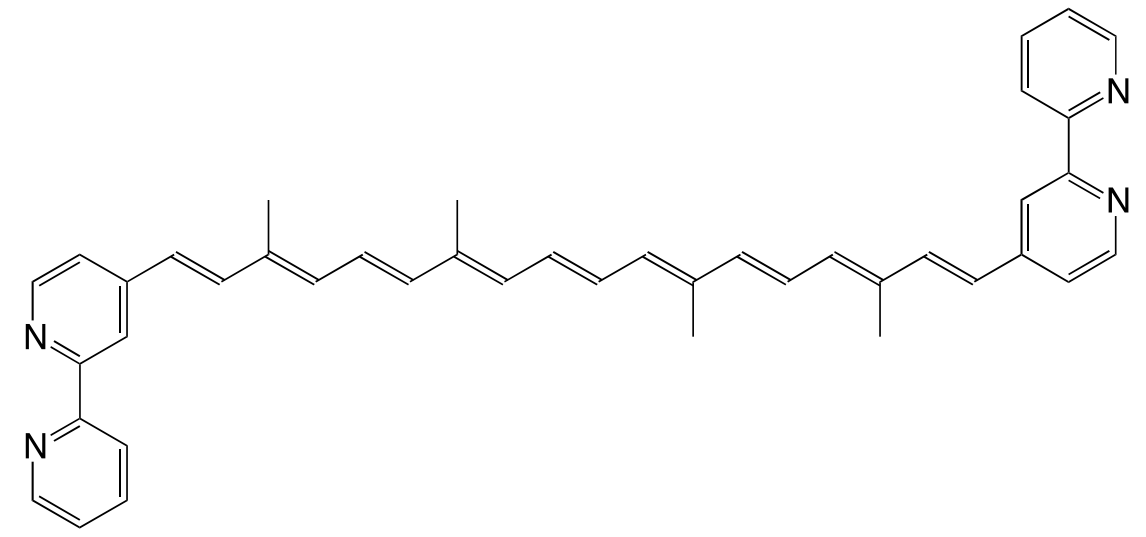

F. Effenberger, M. Wezstein, Synthesis 2001, 1368

$27 \odot \mathrm{N}$ astacene bisphenazine

$\mathrm{C}_{52} \mathrm{H}_{56} \mathrm{~N}_{4}$<smiles>CC1=C(/C=C/C(C)=C/C=C/C=C(C)/C=C/C=C(C)/C=C/C=C(C)/C=C/C2=C(C)c3nc4ccccc4nc3CC2(C)C)C(C)(C)Cc2nc3ccccc3nc21</smiles>

P. Karrer, L. Loewe, Helv. Chim. Acta 1934, 17, 745

S. Hertzberg, S. Liaaen-Jensen, C.R. Enzell, G.W. Francis, Acta Chem. Scand. 1969, 23, 3290 
$28 \odot \mathrm{N}$ violerythrin bisquinoxaline

$\mathrm{C}_{50} \mathrm{H}_{52} \mathrm{~N}_{4}$

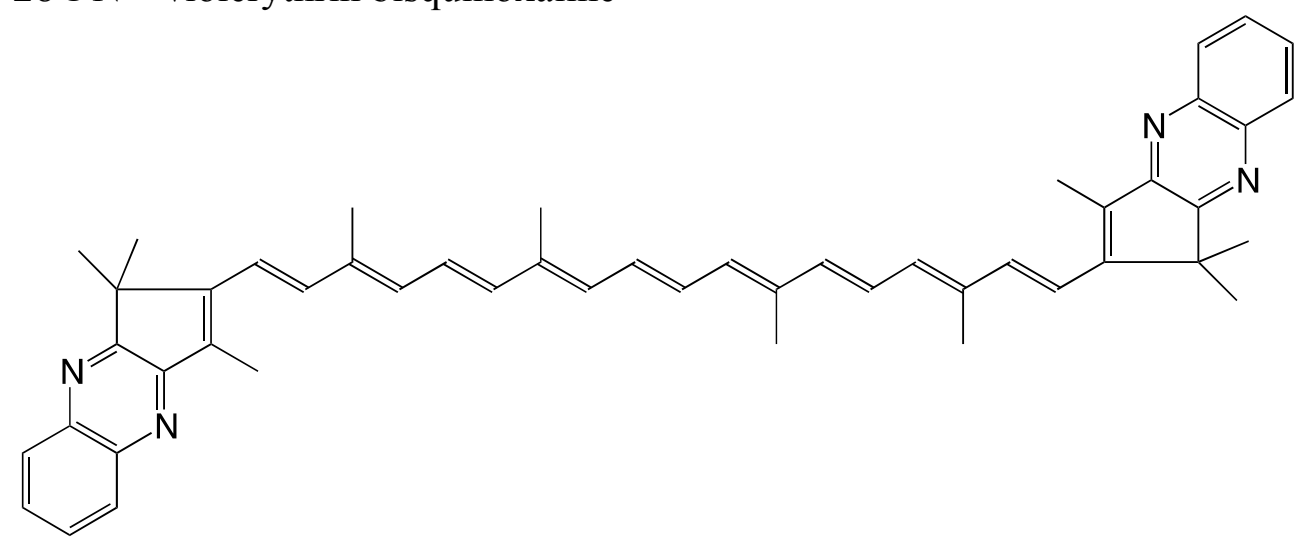

S. Hertzberg, S. Liaaen-Jensen, C.R. Enzell, G.W. Francis, Acta Chem. Scand. 1969, 23, 3290

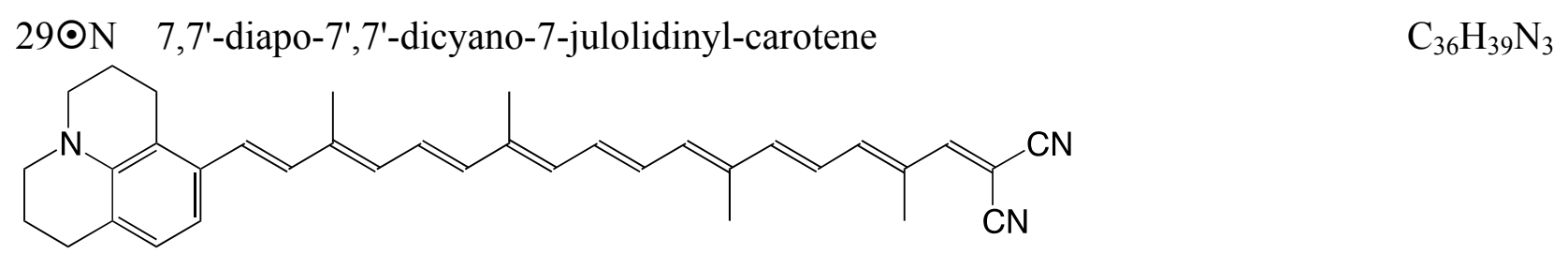

M. Blanchard-Desce, J.M. Lehn, M. Barzoukas, I. Ledoux, J. Zyss, Chem. Phys. 1994, 181, 281

$30 \odot \mathrm{N} \quad$ 7'-apo-3-cyano-7'-julolidinyl- $\phi$-carotene

$\mathrm{C}_{41} \mathrm{H}_{44} \mathrm{~N}_{2}$<smiles>CC(/C=C/C=C(C)/C=C/c1ccc(C#N)cc1)=C\C=C\C=C(C)\C=C\C=C(C)\C=C\c1ccc2c3c1CCCN3CCC2</smiles>

M. Blanchard-Desce, J.M. Lehn, M. Barzoukas, I. Ledoux, J. Zyss, Chem. Phys. 1994, 181, 281

$31 \odot \mathrm{N} \quad 7$ '-apo-7'-julolidinyl-3-nitro- $\phi$-carotene

$\mathrm{C}_{40} \mathrm{H}_{44} \mathrm{~N}_{2} \mathrm{O}_{2}$<smiles>CC(/C=C/C=C/C(C)=C/C=C/C(C)=C/c1ccc([N+](=O)[O-])cc1)=C\C=C\C(C)=C\C=C\c1ccc2c3c1CCCN3CCC2</smiles>

M. Blanchard-Desce, J.M. Lehn, M. Barzoukas, I. Ledoux, J. Zyss, Chem. Phys. 1994, 181, 281 
$32 \odot \mathrm{N}$ diterpyridine carotenoid

$\mathrm{C}_{52} \mathrm{H}_{46} \mathrm{~N}_{6}$<smiles>CC(/C=C/C=C(C)/C=C/C=C(C)/C=C/c1cc(-c2ccccn2)nc(-c2ccccn2)c1)=C\C=C\C=C(C)\C=C\C1=CC(c2ccccn2)NC(c2ccccn2)=C1</smiles>

G. Pickaert, R. Ziessel, Tetrahedron Lett. 1998, 39, 3497

$1 \odot \mathrm{S} \quad 3,4,3$ ',4',-tetrahydrospirilloxanthin-20-(1,3-dithiolane)

$\mathrm{C}_{44} \mathrm{H}_{66} \mathrm{O}_{2} \mathrm{~S}_{2}$<smiles>COC(C)(C)CCC/C(C)=C/C=C/C(C)=C/C=C/C(=C/C=C/C=C(C)/C=C/C=C(C)/C=C/C=C(\C)CCCC(C)(C)OC)C1SCCS1</smiles>

A.J. Aasen, S. Liaaen Jensen, Acta Chem. Scand. 1967, 21, 2185

$2 \odot \mathrm{S}(=1 \mathrm{Si}) \quad$ 7,5'-diapo-7-thienyl-carotene-5'-triethoxysilane

$\mathrm{C}_{34} \mathrm{H}_{46} \mathrm{O}_{3} \mathrm{SSi}$<smiles>CCO[Si](/C=C/C=C/C(C)=C/C=C/C(C)=C/C=C/C=C(C)/C=C/C=C(C)/C=C/c1cccs1)(OCC)OCC</smiles>

F. Effenberger, M. Wezstein, Synthesis 2001, 1368

$3 \odot \mathrm{S} \quad$ 7,7'-diapo-bis(2-thienyl)-carotene<smiles>CC(/C=C/C=C(C)/C=C/C=C(C)/C=C/c1cccs1)=C\C=C\C(C)=C\C=C\c1cccs1</smiles>

$\mathrm{C}_{30} \mathrm{H}_{32} \mathrm{~S}_{2}$

H.R. Brahmana, K. Katsuyama, J. Inaga, T. Katsuki, M. Yamaguchi, Tetrahedron Lett. 1981, 22, 1695 F. Effenberger, M. Wezstein, Synthesis 2001, 1368 
$4 \odot \mathrm{S} \quad$ 7,7'-diapo-bis(3-thienyl)-carotene

$\mathrm{C}_{30} \mathrm{H}_{32} \mathrm{~S}_{2}$<smiles>CC(/C=C/C=C(C)/C=C/c1ccsc1)=C\C=C\C=C\C(C)=C\C=C\C(C)=C\c1ccsc1</smiles>

H.R. Brahmana, K. Katsuyama, J. Inaga, T. Katsuki, M. Yamaguchi, Tetrahedron Lett. 1981, 22, 1695

$5 \odot \mathrm{S}$ 7',8-diapo-7',7'-dicyano-8-(benzodithiol-2-ylidene)-carotene

$\mathrm{C}_{30} \mathrm{H}_{28} \mathrm{~N}_{2} \mathrm{~S}_{2}$<smiles>C/C(C=C1Sc2ccccc2S1)=C\C=C\C=C(C)\C=C\C=C(C)\C=C(/C)C=C(C#N)C#N</smiles>

M. Blanchard-Desce, I. Ledoux, J. Malthête, J. Zyss, J. Chem. Soc., Chem. Commun. 1988, 737

$6 \odot \mathrm{S} \quad 8^{\prime}$-apo-8'-(benzodithiol-2-ylidene)-3-cyano- $\phi$-carotene

$\mathrm{C}_{35} \mathrm{H}_{33} \mathrm{NS}_{2}$<smiles>C/C=C/C=C(C)/C=C/C=C/C(C)=C/C=C/C(C)=C/C=C/C=C(C)/C=C/c1ccc(C#N)cc1</smiles>

M. Blanchard-Desce, I. Ledoux, J. Malthête, J. Zyss, J. Chem. Soc., Chem. Commun. 1988, 737

$7 \odot S$ 8'-apo-8'-(benzodithiol-2-ylidene)-3-nitro- $\phi$-carotene $\mathrm{C}_{34} \mathrm{H}_{33} \mathrm{NO}_{2} \mathrm{~S}_{2}$<smiles>C/C(C=C1Sc2ccccc2S1)=C\C=C\C(C)=C\C=C\C=C\C(C)=C\C=C\C(C)=C\c1ccc([N+](=O)[O-])cc1</smiles>

M. Blanchard-Desce, I. Ledoux, J. Malthête, J. Zyss, J. Chem. Soc., Chem. Commun. 1988, 737

$1 \odot \mathrm{N}, \mathrm{S} \quad 7,8^{\prime}$-diapo-8'-(benzodithiol-2-ylidene)-7-(4-pyridyl)-carotene<smiles>C/C(C=C1Sc2ccccc2S1)=C\C=C\C(C)=C\C=C\C(C)=C\C=C\C(C)=C\c1ccncc1</smiles>

M. Blanchard-Desce, I. Ledoux, J. Malthête, J. Zyss, J. Chem. Soc., Chem. Commun. 1988, 737 
$2 \odot \mathrm{N}, \mathrm{S} \quad$ 7,8'-diapo-8'-(benzodithiol-2-ylidene)-7-(4-pyridinium)-carotene iodide

$\mathrm{C}_{34} \mathrm{H}_{36} \mathrm{INS}_{2}$<smiles>C/C(C=C1Sc2ccccc2S1)=C\C=C\C(C)=C\C=C\C=C(C)\C=C\C=C(C)\C=C\c1cc[n+](C)cc1</smiles>

M. Blanchard-Desce, I. Ledoux, J. Malthête, J. Zyss, J. Chem. Soc., Chem. Commun. 1988, 737

$3 \odot \mathrm{N}, \mathrm{S} \quad \mathrm{C} 30$-aldehyde rhodanine, 8 '-apo- $\beta$-carotenyliden-rhodanine

$\mathrm{C}_{33} \mathrm{H}_{41} \mathrm{NOS}_{2}$<smiles>CC1=C(/C=C/C(C)=C/C=C/C(C)=C/C=C/C=C(C)/C=C/C=C2/SC(=S)NC2=O)C(C)(C)CCC1</smiles>

H. Hegedus, US3071583, 1963

H. Thommen, Int. Z. Vitaminforsch. 1967, 37, 175

Sample Availability: Not available.

(C) 2012 by the authors; licensee MDPI, Basel, Switzerland. This article is an open access article distributed under the terms and conditions of the Creative Commons Attribution license (http://creativecommons.org/licenses/by/3.0/). 